\title{
Gaussian Robust Sequential and Predictive Coding
}

\author{
Lin Song, Jun Chen, Member, IEEE, Jia Wang, and Tie Liu
}

\begin{abstract}
We introduce two new source coding problems: robust sequential coding and robust predictive coding. For the Gauss-Markov source model with the mean squared error distortion measure, we characterize certain supporting hyperplanes of the rate region of these two coding problems. Our investigation also reveals an information-theoretic minimax theorem and the associated extremal inequalities.
\end{abstract}

Index Terms-Extremal inequality, Gauss-Markov source, minimax theorem, predictive coding, saddle point, sequential coding.

\section{INTRODUCTION}

$\mathbf{T}$ HE sequential coding problem was first introduced by Viswanathan and Berger in [1] (see also [2] for a closely related problem). Due to its potential relevance to video coding applications, this problem has received renewed interests in recent years [3], [4]. In a sequential coding system, $L$ sources $X_{1}, \ldots, X_{L}$, each representing a video frame, are encoded and decoded in a causal manner, where Encoder $i$ has access to $X_{1}, \ldots, X_{i}, i=1, \ldots, L$, and the decoder reconstructs $X_{i}$ based on the outputs from the first $i$ encoders, $i=1, \ldots, L$. If Encoder $i$ is only allowed to have access to $X_{i}$ as well as the outputs from the first $i-1$ encoders (if $i \geq 2$ ), then the resulting problem is known as predictive coding. It is shown in [5] that the rate regions of these two coding problems are identical if $X_{1} \leftrightarrow X_{2} \leftrightarrow \cdots \leftrightarrow X_{L}$ form a Markov chain. ${ }^{1}$ Note that this Markov chain condition is trivially satisfied when $L=2$.

The existing coding schemes for sequential coding and predictive coding rely critically on the assumption that the decoder has access to the first $i$ encoded frames (i.e., the outputs from the first $i$ encoders) when reconstructing the $i$ th frame (i.e., $X_{i}$ ). As a consequence, these schemes are vulnerable to the loss of encoded frames at the decoder end. Motivated by this observation, we introduce a robust version of these two coding problems.

Manuscript received April 07, 2012; accepted October 29, 2012. Date of publication February 07, 2013; date of current version May 15, 2013. J. Chen was supported in part by an Early Researcher Award from the Province of Ontario and in part by the Natural Science and Engineering Research Council of Canada under a Discovery Grant. J. Wang was supported in part by the National Natural Science Foundation of China under Grants 61271221 and U1201255 and in part by the National Key Technology R\&D Program of China under Grant 2013BAH53F04. T. Liu was supported in part by the National Science Foundation under Grant CCF-09-16867. This paper was presented in part at the 2012 IEEE International Symposium on Information Theory.

L. Song and J. Chen are with the Department of Electrical and Computer Engineering, McMaster University, Hamilton, ON L8S 4K1, Canada (e-mail: songlin@grads.ece.mcmaster.ca; junchen@ece.mcmaster.ca).

J. Wang is with the Department of Electronic Engineering, and the Institute of Image Communication and Network Engineering, Shanghai Jiao Tong University, Shanghai 200240, China (e-mail: jiawang@sjtu.edu.cn).

T. Liu is with the Department of Electrical and Computer Engineering, Texas A\&M University, College Station, TX 77843 USA (e-mail: tieliu@tamu.edu).

Communicated by T. Weissman, Associate Editor for Shannon Theory.

Color versions of one or more of the figures in this paper are available online at http://ieeexplore.ieee.org.

Digital Object Identifier 10.1109/TIT.2013.2245720

${ }^{1}$ This result is also implied by [2, Th. 1].
Specifically, we require that the reconstruction of the $i$ th frame has to meet a certain fidelity constraint even when the decoder only has access to the output from the $i$ th encoder. This formulation is also applicable to the scenario where the encoded frames are to be decoded by two types of decoders: one has the capability of using multiple encoded frames to reconstruct a target frame, while the other can only perform the reconstruction operation based on a single encoded frame (due to storage or complexity constraints).

The remainder of this paper is organized as follows. We state our main results in Section II; these results provide a partial characterization of the rate region of robust sequential coding and robust predictive coding for the Gauss-Markov source model under the mean squared error distortion constraint. The proofs of these results are given in Sections III-V. It is shown in Section VI that our main results can be viewed as a manifestation of an information-theoretic minimax theorem. Section VII contains an explicit characterization of the minimum sum rate for a special class of sources and distortion constraints. We provide a detailed discussion of the proposed robust predictive coding scheme in Section VIII and conclude this paper in Section IX.

For any random object $W$ and $1 \times n$ random vector $X^{n}$, we define $\sigma_{X^{n}}^{2}=\frac{1}{n} \mathbb{E}\left[X^{n}\left(X^{n}\right)^{T}\right]$ and $\sigma_{X^{n} \mid W}^{2}=\sigma_{X^{n}-\mathbb{E}\left[X^{n} \mid W\right]}^{2}$. Throughout this paper, the logarithm function is to base $e$.

\section{MAIN RESUltS}

Consider $L$ sources $X_{1}, \ldots, X_{L}$ with joint distribution $p\left(x_{1}, \ldots, x_{L}\right)$. Let $\left\{\left(X_{1 j}, \ldots, X_{L j}\right)\right\}_{j=1}^{\infty}$ be i.i.d. copies of $\left(X_{1}, \ldots, X_{L}\right)$. Let $w_{i}: \mathcal{X}_{i} \times \hat{\mathcal{X}}_{i} \rightarrow[0, \infty)$ be a distortion measure, where $\mathcal{X}_{i}$ and $\hat{\mathcal{X}}_{i}$ are, respectively, the source alphabet (of $X_{i}$ ) and reconstruction alphabet, $i=1, \ldots, L$.

Definition 1: A rate vector $\bar{R} \triangleq\left(R_{1}, \ldots, R_{L}\right)$ is said to be achievable with a sequential coding system subject to hierarchical distortion constraint $\bar{d} \triangleq\left(d_{1}, \ldots, d_{L}\right)$ and individual distortion constraint $\bar{\delta} \triangleq\left(\delta_{2}, \ldots, \delta_{L}\right)$ if for every $\epsilon>0$, there exist encoding functions $f_{i}^{(n)}: \mathcal{X}_{1}^{n} \times \cdots \times \mathcal{X}_{i}^{n} \rightarrow \mathcal{C}_{i}, i=1, \ldots, L$, and decoding functions $\tilde{g}_{i}^{(n)}: \mathcal{C}_{1} \times \cdots \times \mathcal{C}_{i} \rightarrow \hat{\mathcal{X}}_{i}^{n}, i=1, \ldots, L$, and $\hat{g}_{i}^{(n)}: \mathcal{C}_{i} \rightarrow \hat{\mathcal{X}}_{i}^{n}, i=2, \ldots, L$, such that

$$
\begin{aligned}
& \frac{1}{n} \log \left|\mathcal{C}_{i}\right| \leq R_{i}+\epsilon, \quad i=1, \ldots, L \\
& \mathbb{E}\left[\frac{1}{n} \sum_{j=1}^{n} w_{i}\left(X_{i j}, \tilde{X}_{i j}\right)\right] \leq d_{i}+\epsilon, \quad i=1, \ldots, L \\
& \mathbb{E}\left[\frac{1}{n} \sum_{j=1}^{n} w_{i}\left(X_{i j}, \hat{X}_{i j}\right)\right] \leq \delta_{i}+\epsilon, \quad i=2, \ldots, L
\end{aligned}
$$

where $\tilde{X}_{i}^{n}=\tilde{g}_{i}^{(n)}\left(C_{1}, \ldots, C_{i}\right), i=1, \ldots, L$, and $\hat{X}_{i}^{n}=$ $\hat{g}_{i}^{(n)}\left(C_{i}\right), i=2, \ldots, L$, with $C_{i}=f_{i}^{(n)}\left(X_{1}^{n}, \ldots, X_{i}^{n}\right), i=$ 


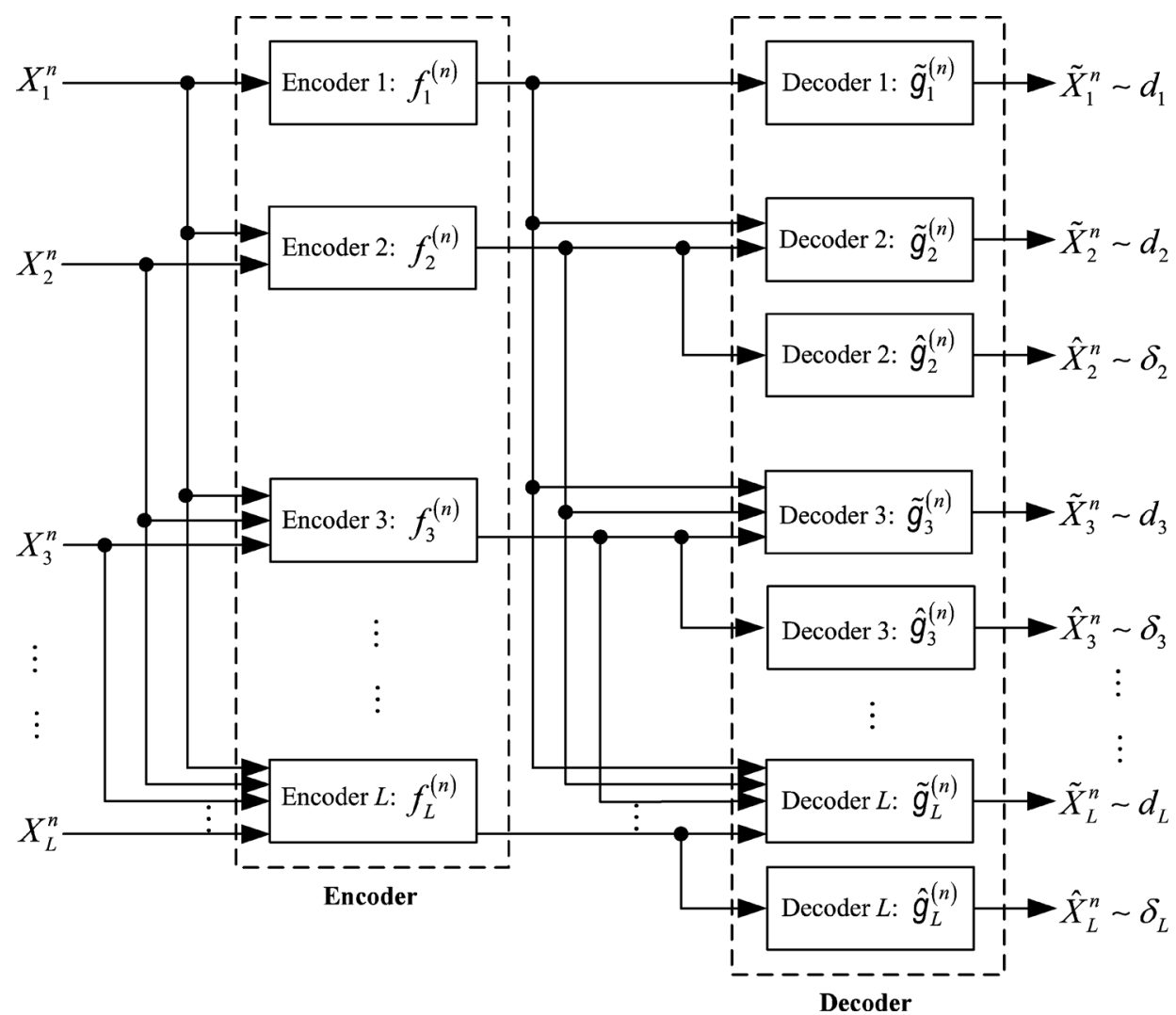

Fig. 1. Robust sequential coding with hierarchical and individual distortion constraints.

$1, \ldots, L$. The rate region $\mathcal{R}_{S}(\bar{d}, \bar{\delta})$ is the set of all the rate vectors achievable with a sequential coding system subject to hierarchical distortion constraint $\bar{d}$ and individual distortion constraint $\bar{\delta}$.

A system diagram of robust sequential coding with hierarchical and individual distortion constraints can be found in Fig. 1.

Definition 2: A rate vector $\bar{R} \triangleq\left(R_{1}, \ldots, R_{L}\right)$ is said to be achievable with a predictive coding system subject to hierarchical distortion constraint $\bar{d} \triangleq\left(d_{1}, \ldots, d_{L}\right)$ and individual distortion constraint $\bar{\delta} \triangleq\left(\delta_{2}, \ldots, \delta_{L}\right)$ if for every $\epsilon>0$, there exist encoding functions $f_{1}^{(n)}: \mathcal{X}_{1}^{n} \rightarrow \mathcal{C}_{1}$ and $f_{i}^{(n)}: \mathcal{C}_{1} \times \cdots \times$ $\mathcal{C}_{i-1} \times \mathcal{X}_{i}^{n} \rightarrow \mathcal{C}_{i}, i=2, \ldots, L$, and decoding functions $\tilde{g}_{i}^{(n)}:$ $\mathcal{C}_{1} \times \cdots \times \mathcal{C}_{i} \rightarrow \hat{\mathcal{X}}_{i}^{n}, i=1, \ldots, L$, and $\hat{g}_{i}^{(n)}: \mathcal{C}_{i} \rightarrow \hat{\mathcal{X}}_{i}^{n}$, $i=2, \ldots, L$, such that

$$
\begin{aligned}
& \frac{1}{n} \log \left|\mathcal{C}_{i}\right| \leq R_{i}+\epsilon, \quad i=1, \ldots, L \\
& \mathbb{E}\left[\frac{1}{n} \sum_{j=1}^{n} w_{i}\left(X_{i j}, \tilde{X}_{i j}\right)\right] \leq d_{i}+\epsilon, \quad i=1, \ldots, L \\
& \mathbb{E}\left[\frac{1}{n} \sum_{j=1}^{n} w_{i}\left(X_{i j}, \hat{X}_{i j}\right)\right] \leq \delta_{i}+\epsilon, \quad i=2, \ldots, L
\end{aligned}
$$

where $\tilde{X}_{i}^{n}=\tilde{g}_{i}^{(n)}\left(C_{1}, \ldots, C_{i}\right), i=1, \ldots, L$, and $\hat{X}_{i}^{n}=\hat{g}_{i}^{(n)}\left(C_{i}\right), i=2, \ldots, L$, with $C_{1}=f_{1}^{(n)}\left(X_{1}^{n}\right)$ and $C_{i}=f_{i}^{(n)}\left(C_{1}, \ldots, C_{i-1}, X_{i}^{n}\right), i=2, \ldots, L$. The rate region $\mathcal{R}_{P}(\bar{d}, \bar{\delta})$ is the set of all the rate vectors achievable with a predictive coding system subject to hierarchical distortion constraint $\bar{d}$ and individual distortion constraint $\bar{\delta}$.
A system diagram of robust predictive coding with hierarchical and individual distortion constraints can be found in Fig. 2.

In this study, we focus on the special case where $X_{1} \leftrightarrow X_{2} \leftrightarrow$ $\cdots \leftrightarrow X_{L}$ form a Gauss-Markov chain. With no essential loss of generality, we assume $X_{i+1}=X_{i}+\Delta_{i}, i=1, \ldots, L-1$, where $X_{1}, \Delta_{1}, \ldots, \Delta_{L-1}$ are mutually independent zero-mean Gaussian random variables with $\sigma_{X_{1}}^{2}>0$ and $\sigma_{\Delta_{i}}^{2}>0, i=$ $1, \ldots, L-1$. Furthermore, we use the mean squared error as the distortion measure, i.e., $w_{i}\left(x_{i}, \hat{x}_{i}\right)=\left(x_{i}-\hat{x}_{i}\right)^{2}$ for all $x_{i} \in \mathbb{R}$ and $\hat{x}_{i} \in \mathbb{R}, i=1, \ldots, L$. Note that in this setting, there is no loss of optimality in assuming $\tilde{g}_{i}^{(n)}\left(C_{1}, \ldots, C_{i}\right)=$ $\mathbb{E}\left[X_{i}^{n} \mid C_{1}, \ldots, C_{i}\right], i=1, \ldots, L$, and $\hat{g}_{i}^{(n)}\left(C_{i}\right)=\mathbb{E}\left[X_{i}^{n} \mid C_{i}\right]$, $i=2, \ldots, L$. As a consequence, (1) and (3) can be rewritten as

$$
\sigma_{X_{i}^{n} \mid C_{1}, \ldots, C_{i}}^{2} \leq d_{i}+\epsilon, \quad i=1, \ldots, L .
$$

For the same reason, (2) and (4) can be rewritten as

$$
\sigma_{X_{i}^{n} \mid C_{i}}^{2} \leq \delta_{i}+\epsilon, \quad i=2, \ldots, L
$$

Without loss of generality, we assume $0<d_{i} \leq \sigma_{X_{i}}^{2}, i=$ $1, \ldots, L$, and $0<\delta_{i} \leq \sigma_{X_{i}}^{2}, i=2, \ldots, L$. Since both $\mathcal{R}_{S}(\bar{d}, \bar{\delta})$ and $\mathcal{R}_{P}(\bar{d}, \bar{\delta})$ are closed convex sets, it suffices to characterize their supporting hyperplanes, i.e., to solve the following optimization problems:

$$
\inf _{\bar{R} \in \mathcal{R}_{S}(\bar{d}, \bar{\delta})} \bar{\mu} \bar{R}^{T} \quad \text { and } \quad \inf _{\bar{R} \in \mathcal{R}_{P}(\bar{d}, \bar{\delta})} \bar{\mu} \bar{R}^{T}
$$

where $\bar{\mu}=\left(\mu_{1}, \ldots, \mu_{L}\right)$ with $\mu_{i} \geq 0, i=1, \ldots, L$. In view of the fact that $\mathcal{R}_{P}(\bar{d}, \bar{\delta}) \subseteq \mathcal{R}_{S}(\bar{d}, \bar{\delta})$, we must have

$$
\inf _{\bar{R} \in \mathcal{R}_{S}(\bar{d}, \bar{\delta})} \bar{\mu} \bar{R}^{T} \leq \inf _{\bar{R} \in \mathcal{R}_{P}(\bar{d}, \bar{\delta})} \bar{\mu} \bar{R}^{T} .
$$




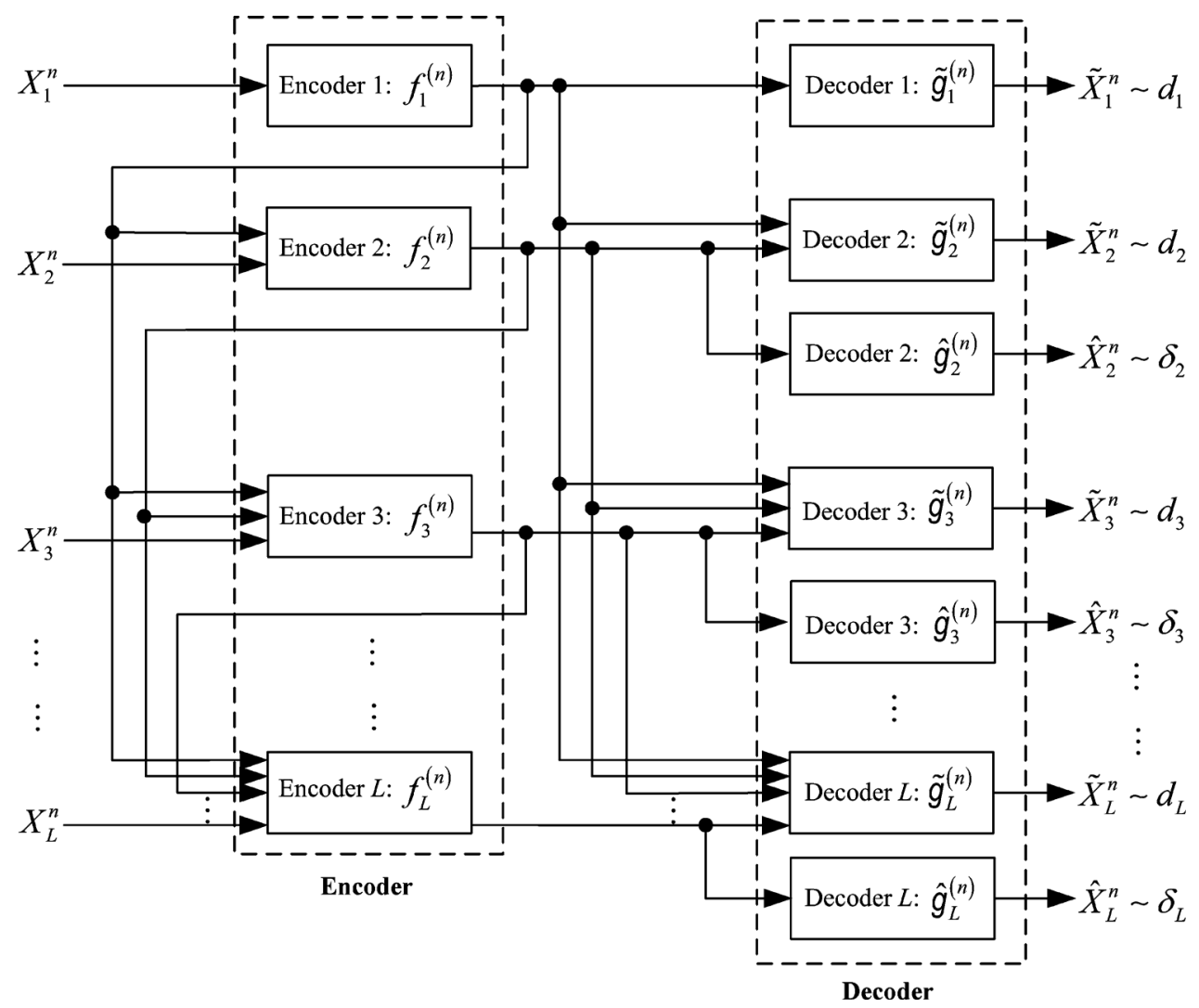

Fig. 2. Robust predictive coding with hierarchical and individual distortion constraints.

To state the main results of this paper, we need to define the following function:

$$
\begin{aligned}
& \psi(\bar{\mu}, \bar{\gamma}, \bar{\delta}, \bar{\theta}) \\
& =\sum_{i=2}^{L} \frac{\mu_{i}}{2} \log \left(\frac{\sigma_{X_{i}}^{4}\left(\gamma_{i-1}+\sigma_{\Delta_{i-1}}^{2}\right)}{\left(\left(\sigma_{X_{i}}^{2}-\theta_{i-1}\right)\left(\gamma_{i-1}+\sigma_{\Delta_{i-1}}^{2}\right)+\sigma_{X_{i}}^{2} \theta_{i-1}\right)}\right. \\
& \left.\quad \times \frac{\left(\left(\sigma_{X_{i}}^{2}-\theta_{i-1}\right) \gamma_{i}+\sigma_{X_{i}}^{2} \theta_{i-1}\right)}{\left(\left(\sigma_{X_{i}}^{2}-\theta_{i-1}\right) \delta_{i}+\sigma_{X_{i}}^{2} \theta_{i-1}\right) \gamma_{i}}\right)+\frac{\mu_{1}}{2} \log \left(\frac{\sigma_{X_{1}}^{2}}{\gamma_{1}}\right)
\end{aligned}
$$

where $\bar{\gamma}=\left(\gamma_{1}, \ldots, \gamma_{L}\right)$ and $\bar{\theta}=\left(\theta_{1}, \ldots, \theta_{L-1}\right)$. Furthermore, let

$$
\begin{aligned}
& \kappa_{l}(\bar{\mu}, \bar{d}, \bar{\delta}) \\
& =\sup _{\theta_{i} \in\left(0, \sigma_{X_{i+1}}^{2}\right), i=1, \ldots, L-1} \min _{\gamma_{i} \in\left[0, d_{i}\right], i=1, \ldots, L} \psi(\bar{\mu}, \bar{\gamma}, \bar{\delta}, \bar{\theta}) \\
& \kappa_{u}(\bar{\mu}, \bar{d}, \bar{\delta}) \\
& =\inf _{\gamma_{i} \in\left(0, d_{i}\right), i=1, \ldots, L} \max _{\theta_{i} \in\left[0, \sigma_{X_{i+1}}^{2}\right], i=1, \ldots, L-1} \psi(\bar{\mu}, \bar{\gamma}, \bar{\delta}, \bar{\theta}) .
\end{aligned}
$$

Theorem 1: For $\bar{\mu}$ with $\mu_{1} \geq \cdots \geq \mu_{L} \geq 0$

$$
\inf _{\bar{R} \in \mathcal{R}_{S}(\bar{d}, \bar{\delta})} \bar{\mu} \bar{R}^{T} \geq \kappa_{l}(\bar{\mu}, \bar{d}, \bar{\delta})
$$

Theorem 2: For $\bar{\mu}$ with $\mu_{i} \geq 0, i=1, \ldots, L$,

$$
\inf _{\bar{R} \in \mathcal{R}_{P}(\bar{d}, \bar{\delta})} \bar{\mu} \bar{R}^{T} \leq \kappa_{u}(\bar{\mu}, \bar{d}, \bar{\delta}) .
$$

Theorem 3: For $\bar{\mu}$ with $\mu_{1} \geq \cdots \geq \mu_{L} \geq 0$

$$
\kappa_{l}(\bar{\mu}, \bar{d}, \bar{\delta})=\kappa_{u}(\bar{\mu}, \bar{d}, \bar{\delta}) .
$$

The proofs of Theorems 1-3 are given in Sections III-V, respectively. These theorems together with (5) lead to the following result, which provides a characterization of certain supporting hyperplanes of $\mathcal{R}_{S}(\bar{d}, \bar{\delta})$ and $\mathcal{R}_{P}(\bar{d}, \bar{\delta})$; in particular, setting $\mu_{1}=\cdots=\mu_{L}=1$ gives the minimum sum rate of these two rate regions.

Theorem 4: For $\bar{\mu}$ with $\mu_{1} \geq \cdots \geq \mu_{L} \geq 0$

$$
\inf _{\bar{R} \in \mathcal{R}_{S}(\bar{d}, \bar{\delta})} \bar{\mu}^{T}=\inf _{\bar{R} \in \mathcal{R}_{P}(\bar{d}, \bar{\delta})} \bar{\mu}^{T}=\kappa_{l}(\bar{\mu}, \bar{d}, \bar{\delta})=\kappa_{u}(\bar{\mu}, \bar{d}, \bar{\delta}) .
$$

For the special case $L=2$, one can verify that $\kappa_{l}(\bar{\mu}, \bar{d}, \bar{\delta})$ and $\kappa_{u}(\bar{\mu}, \bar{d}, \bar{\delta})$ have the following explicit expression (when $\left.\mu_{1} \geq \mu_{2} \geq 0\right)$ :

$$
\begin{aligned}
& \kappa_{l}(\bar{\mu}, \bar{d}, \bar{\delta}) \\
& =\kappa_{u}(\bar{\mu}, \bar{d}, \bar{\delta}) \\
& =\left\{\begin{array}{c}
\frac{\mu_{1}}{2} \log \left(\frac{\sigma_{X_{1}}^{2}}{d_{1}}\right)+\frac{\mu_{2}}{2} \log \left(\frac{d_{1}+\sigma_{\Delta_{1}}^{2}}{d_{2}}\right), \\
d_{2} \leq d_{1}+\sigma_{\Delta_{1}}^{2}+\delta_{2}-\sigma_{X_{2}}^{2} \\
\frac{\mu_{1}}{2} \log \left(\frac{\sigma_{X_{1}}^{2}}{d_{1}}\right)+\frac{\mu_{2}}{2} \log \left(\frac{\sigma_{X_{2}}^{2}}{\delta_{2}}\right), \\
d_{2} \geq\left(\left(d_{1}+\sigma_{\Delta_{1}}^{2}\right)^{-1}+\delta_{2}^{-1}-\sigma_{X_{2}}^{-2}\right)^{-1} \\
\frac{\mu_{1}}{2} \log \left(\frac{\sigma_{X_{1}}^{2}}{d_{1}}\right)+\frac{\mu_{2}}{2} \log \left(\frac{\left(d_{1}+\sigma_{\Delta_{1}}^{2}\right)\left(\sigma_{X_{2}}^{2}-d_{2}\right)^{2}}{d_{2}\left(\left(\sigma_{X_{2}}^{2}-d_{2}\right)^{2}-\left(\varrho_{1}-\varrho_{2}\right)^{2}\right)}\right), \\
\text { otherwise }
\end{array}\right.
\end{aligned}
$$


where

$$
\begin{aligned}
& \varrho_{1}=\sqrt{\left(\sigma_{X_{2}}^{2}-d_{1}-\sigma_{\Delta_{1}}^{2}\right)\left(\sigma_{X_{2}}^{2}-\delta_{2}\right)} \\
& \varrho_{2}=\sqrt{\left(d_{1}+\sigma_{\Delta_{1}}^{2}-d_{2}\right)\left(\delta_{2}-d_{2}\right)} .
\end{aligned}
$$

Our formulation of robust sequential coding and predictive coding is partly inspired by the classic multiple description problem (see, e.g., [6] and [7]). In fact, the problem treated in [7] can be viewed as a degenerate case of our setting with $\sigma_{\Delta_{i}}^{2}=0, i=1, \ldots, L-1$. It will be seen that such a connection allows us to leverage the techniques developed for the multiple description problem and, albeit somewhat implicitly, provides conceptual guidelines for our analysis. However, a straightforward application of the existing techniques turns out to be insufficient for handling these new problems. Indeed, we need to establish a new extremal inequality for the converse argument (see Section III); the achievability scheme (see Section IV) and the saddle point analysis (see Section V) are also more delicate than their counterparts in [7]. Moreover, the new coding problems possess certain features not found in the multiple description problem; for example, the special case studied in Section VII has no natural counterpart in multiple description coding. Finally and most importantly, the analysis of the new coding problems enables us to extract an information-theoretic minimax theorem which is of interest in its own right (see Section VI).

\section{Proof of Theorem 1}

The following extremal inequality is the main technical ingredient in the proof of Theorem 1. It can be viewed as a generalization of [7, Lemma 1]. The proof is given in Appendix A.

Theorem 5: Let $N_{i}^{n}$ be a zero-mean Gaussian random vector with i.i.d. entries of positive variance $\sigma_{N_{i}}^{2}, i=1,2,3$, where $\sigma_{N_{2}}^{2} \leq \sigma_{N_{3}}^{2}$. Let $\nu_{1}, \nu_{2}, \rho_{1}, \rho_{2}$, and $d$ be arbitrary real numbers satisfying $\nu_{1} \geq \nu_{2} \geq 0$ and $d>0$. Then, for any random vector $S^{n}$ and random object $W$, jointly independent of $\left(N_{1}^{n}, N_{2}^{n}, N_{3}^{n}\right)$, such that $\sigma_{S^{n} \mid W}^{2} \leq d$,

$$
\begin{aligned}
& \nu_{1}\left(h\left(\rho_{1} S^{n}+N_{1}^{n} \mid W\right)-h\left(S^{n} \mid W\right)\right) \\
& \quad-\nu_{2}\left(h\left(\rho_{2} S^{n}+N_{3}^{n} \mid W\right)-h\left(\rho_{2} S^{n}+N_{2}^{n} \mid W\right)\right) \\
& \geq \min _{\gamma \in[0, d]} \frac{\nu_{1} n}{2} \log \left(\frac{\rho_{1}^{2} \gamma+\sigma_{N_{1}}^{2}}{\gamma}\right)-\frac{\nu_{2} n}{2}\left(\frac{\rho_{2}^{2} \gamma+\sigma_{N_{3}}^{2}}{\rho_{2}^{2} \gamma+\sigma_{N_{2}}^{2}}\right) .
\end{aligned}
$$

Now we proceed to prove Theorem 1 . The proof relies on Theorem 5 as well as the techniques developed in [7]-[9]. Given $\bar{R} \in \mathcal{R}_{S}(\bar{d}, \bar{\delta})$, it suffices to show that

$$
\bar{\mu} \bar{R}^{T} \geq \min _{\gamma_{i} \in\left[0, d_{i}\right], i=1, \ldots, L} \psi(\bar{\mu}, \bar{\gamma}, \bar{\delta}, \bar{\theta})
$$

for all $\bar{\theta}$ with $\theta_{i} \in\left(0, \sigma_{X_{i+1}}^{2}\right), i=1, \ldots, L-1$.

By Definition 1 , for every $\epsilon>0$, there exist $L$ encoding functions $f_{i}^{(n)}: \mathbb{R}^{i \times n} \rightarrow \mathcal{C}_{i}, i=1, \ldots, L$, such that

$$
\begin{aligned}
& \frac{1}{n} \log \left|\mathcal{C}_{i}\right| \leq R_{i}+\epsilon, \quad i=1, \ldots, L \\
& \sigma_{X_{i}^{n} \mid C_{1}, \ldots, C_{i}}^{2} \leq d_{i}+\epsilon, \quad i=1, \ldots, L \\
& \sigma_{X_{i}^{n} \mid C_{i}}^{2} \leq \delta_{i}+\epsilon, \quad i=2, \ldots, L
\end{aligned}
$$

where $C_{i}=f_{i}^{(n)}\left(X_{1}^{n}, \ldots, X_{i}^{n}\right), i=1, \ldots, L$. One can readily verify that

$$
\begin{aligned}
& \sum_{i=1}^{L} \mu_{i}\left(R_{i}+\epsilon\right) \\
& \geq \sum_{i=1}^{L} \frac{\mu_{i}}{n} H\left(C_{i}\right) \\
& \geq \frac{\mu_{1}}{n} I\left(X_{1}^{n} ; C_{1}\right)+\sum_{i=2}^{L} \frac{\mu_{i}}{n} I\left(C_{1}, \ldots, C_{i-1}, X_{i}^{n} ; C_{i}\right) \\
& =\frac{\mu_{1}}{n} I\left(X_{1}^{n} ; C_{1}\right)+\sum_{i=2}^{L} \frac{\mu_{i}}{n}\left(I\left(C_{1}, \ldots, C_{i-1} ; C_{i}\right)\right. \\
& \left.\quad \quad+I\left(X_{i}^{n} ; C_{1}, \ldots, C_{i}\right)-I\left(X_{i}^{n} ; C_{1}, \ldots, C_{i-1}\right)\right) .
\end{aligned}
$$

Let $Z_{i}^{n}$ be a zero-mean Gaussian random vector with i.i.d. entries of positive variance $\sigma_{Z_{i}}^{2}, i=1, \ldots, L-1$; moreover, we assume $Z_{i}^{n}$ is independent of $\left(X_{i+1}^{n}, C_{1}, \ldots, C_{i+1}\right), i=$ $1, \ldots, L-1$. Note that

$$
\begin{aligned}
I( & \left(C_{1}, \ldots, C_{i-1} ; C_{i}\right) \\
= & I\left(X_{i}^{n}+Z_{i-1}^{n} ; C_{1}, \ldots, C_{i-1}\right)+I\left(X_{i}^{n}+Z_{i-1}^{n} ; C_{i}\right) \\
& +I\left(C_{1}, \ldots, C_{i-1} ; C_{i} \mid X_{i}^{n}+Z_{i-1}^{n}\right) \\
& -I\left(X_{i}^{n}+Z_{i-1}^{n} ; C_{1}, \ldots, C_{i}\right) \\
\geq & I\left(X_{i}^{n}+Z_{i-1}^{n} ; C_{1}, \ldots, C_{i-1}\right)+I\left(X_{i}^{n}+Z_{i-1}^{n} ; C_{i}\right) \\
& -I\left(X_{i}^{n}+Z_{i-1}^{n} ; C_{1}, \ldots, C_{i}\right), \quad i=2, \ldots, L .
\end{aligned}
$$

Continuing from (7)

$$
\begin{aligned}
& \sum_{i=1}^{L} \mu_{i}\left(R_{i}+\epsilon\right) \\
& \geq \frac{\mu_{1}}{n} I\left(X_{1}^{n} ; C_{1}\right)+\sum_{i=2}^{L} \frac{\mu_{i}}{n}\left(I\left(X_{i}^{n}+Z_{i-1}^{n} ; C_{1}, \ldots, C_{i-1}\right)\right. \\
& \quad+I\left(X_{i}^{n}+Z_{i-1}^{n} ; C_{i}\right)-I\left(X_{i}^{n}+Z_{i-1}^{n} ; C_{1}, \ldots, C_{i}\right) \\
& \left.\quad+I\left(X_{i}^{n} ; C_{1}, \ldots, C_{i}\right)-I\left(X_{i}^{n} ; C_{1}, \ldots, C_{i-1}\right)\right) \\
& =\frac{\mu_{1}}{n} I\left(X_{1}^{n}, C_{1}\right)-\frac{\mu_{2}}{n}\left(I\left(X_{2}^{n} ; C_{1}\right)-I\left(X_{2}^{n}+Z_{1}^{n} ; C_{1}\right)\right) \\
& +\sum_{i=2}^{L-1}\left(\frac { \mu _ { i } } { n } \left(I\left(X_{i}^{n} ; C_{1}, \ldots, C_{i}\right)\right.\right. \\
& \left.\quad-I\left(X_{i}^{n}+Z_{i-1}^{n} ; C_{1}, \ldots, C_{i}\right)\right) \\
& \quad-\frac{\mu_{i+1}}{n}\left(I\left(X_{i+1}^{n} ; C_{1}, \ldots, C_{i}\right)\right. \\
& \left.\left.\quad-I\left(X_{i+1}^{n}+Z_{i}^{n} ; C_{1}, \ldots, C_{i}\right)\right)\right) \\
& +\frac{\mu_{L}}{n}\left(I\left(X_{L}^{n} ; C_{1}, \ldots, C_{L}\right)-I\left(X_{L}^{n}+Z_{L-1}^{n} ; C_{1}, \ldots, C_{L}\right)\right) \\
& \quad+\sum_{i=2}^{L} \frac{\mu_{i}}{n} I\left(X_{i}^{n}+Z_{i-1}^{n} ; C_{i}\right) \\
& =\frac{\mu_{1}}{2} \log \left(2 \pi e \sigma_{X_{1}}^{2}\right)-\frac{\mu_{2}}{2} \log \left(\frac{\sigma_{X_{2}}^{2}}{\sigma_{X_{2}}^{2}+\sigma_{Z_{1}}^{2}}\right)-\frac{\mu_{1}}{n} h\left(X_{1}^{n} \mid C_{1}\right) \\
& \quad-\frac{\mu_{2}}{n}\left(h\left(X_{2}^{n}+Z_{1}^{n} \mid C_{1}\right)-h\left(X_{2}^{n} \mid C_{1}\right)\right) \\
& + \\
& +\sum_{i=2}^{L-1}\left(\frac{\mu_{i}}{2} \log \left(\frac{\sigma_{X_{i}}^{2}}{\sigma_{X_{i}}^{2}+\sigma_{Z_{i-1}}^{2}}\right)\right. \\
& \quad
\end{aligned}
$$




$$
\begin{aligned}
& -\frac{\mu_{i+1}}{2} \log \left(\frac{\sigma_{X_{i+1}}^{2}}{\sigma_{X_{i+1}}^{2}+\sigma_{Z_{i}}^{2}}\right) \\
& +\frac{\mu_{i}}{n}\left(h\left(X_{i}^{n}+Z_{i-1}^{n} \mid C_{1}, \ldots, C_{i}\right)-h\left(X_{i}^{n} \mid C_{1}, \ldots, C_{i}\right)\right) \\
& -\frac{\mu_{i+1}}{n}\left(h\left(X_{i+1}^{n}+Z_{i}^{n} \mid C_{1}, \ldots, C_{i}\right)\right. \\
& \left.\left.-h\left(X_{i+1}^{n} \mid C_{1}, \ldots, C_{i}\right)\right)\right) \\
+ & \frac{\mu_{L}}{2} \log \left(\frac{\sigma_{X_{L}}^{2}}{\sigma_{X_{L}}^{2}+\sigma_{Z_{L-1}}^{2}}\right) \\
+ & \frac{\mu_{L}}{n}\left(h\left(X_{L}^{n}+Z_{L-1}^{n} \mid C_{1}, \ldots, C_{L}\right)-h\left(X_{L}^{n} \mid C_{1}, \ldots, C_{L}\right)\right) \\
+ & \sum_{i=2}^{L}\left(\frac{\mu_{i}}{2} \log \left(2 \pi e\left(\sigma_{X_{i}}^{2}+\sigma_{Z_{i-1}}^{2}\right)\right)\right. \\
& \left.-\frac{\mu_{i}}{n} h\left(X_{i}^{n}+Z_{i-1}^{n} \mid C_{i}\right)\right)
\end{aligned}
$$

where (9) is due to (8). Note that

$$
\begin{aligned}
- & \mu_{1} h\left(X_{1}^{n} \mid C_{1}\right)-\mu_{2}\left(h\left(X_{2}^{n}+Z_{1}^{n} \mid C_{1}\right)-h\left(X_{2}^{n} \mid C_{1}\right)\right) \\
= & -\mu_{1} h\left(X_{1}^{n} \mid C_{1}\right)-\mu_{2}\left(h\left(X_{1}^{n}+\Delta_{1}^{n}+Z_{1}^{n} \mid C_{1}\right)\right. \\
& \left.-h\left(X_{1}^{n}+\Delta_{1}^{n} \mid C_{1}\right)\right) \\
\geq & \frac{-\mu_{1} n}{2} \log \left(2 \pi e\left(d_{1}+\epsilon\right)\right) \\
& -\frac{\mu_{2} n}{2} \log \left(\frac{d_{1}+\epsilon+\sigma_{\Delta_{1}}^{2}+\sigma_{Z_{1}}^{2}}{d_{1}+\epsilon+\sigma_{\Delta_{1}}^{2}}\right) \\
= & \min _{\gamma_{1} \in\left[0, d_{1}+\epsilon\right]} \frac{-\mu_{1} n}{2} \log \left(2 \pi e \gamma_{1}\right) \\
& \quad-\frac{\mu_{2} n}{2} \log \left(\frac{\gamma_{1}+\sigma_{\Delta_{1}}^{2}+\sigma_{Z_{1}}^{2}}{\gamma_{1}+\sigma_{\Delta_{1}}^{2}}\right)
\end{aligned}
$$

where (11) follows from Lemma 9 in Appendix A. Moreover, we have

$$
\begin{aligned}
\mu_{i}( & \left.h\left(X_{i}^{n}+Z_{i-1}^{n} \mid C_{1}, \ldots, C_{i}\right)-h\left(X_{i}^{n} \mid C_{1}, \ldots, C_{i}\right)\right) \\
& -\mu_{i+1}\left(h\left(X_{i+1}^{n}+Z_{i}^{n} \mid C_{1}, \ldots, C_{i}\right)\right. \\
& \left.\quad-h\left(X_{i+1}^{n} \mid C_{1}, \ldots, C_{i}\right)\right) \\
= & \mu_{i}\left(h\left(X_{i}^{n}+Z_{i-1}^{n} \mid C_{1}, \ldots, C_{i}\right)-h\left(X_{i}^{n} \mid C_{1}, \ldots, C_{i}\right)\right) \\
& -\mu_{i+1}\left(h\left(X_{i}^{n}+\Delta_{i}^{n}+Z_{i}^{n} \mid C_{1}, \ldots, C_{i}\right)\right. \\
& \left.\quad-h\left(X_{i}^{n}+\Delta_{i}^{n} \mid C_{1}, \ldots, C_{i}\right)\right) \\
\geq & \min _{\gamma_{i} \in\left[0, d_{i}+\epsilon\right]} \frac{\mu_{i} n}{2} \log \left(\frac{\gamma_{i}+\sigma_{Z_{i-1}}^{2}}{\gamma_{i}}\right) \\
& \quad-\frac{\mu_{i+1} n}{2} \log \left(\frac{\gamma_{i}+\sigma_{\Delta_{i}}^{2}+\sigma_{Z_{i}}^{2}}{\gamma_{i}+\sigma_{\Delta_{i}}^{2}}\right), \quad i=2, \ldots, L-1
\end{aligned}
$$

where (13) follows from Theorem 5. Note that

$$
\begin{aligned}
& h\left(X_{L}^{n}+Z_{L-1}^{n} \mid C_{1}, \ldots, C_{L}\right)-h\left(X_{L}^{n} \mid C_{1}, \ldots, C_{L}\right) \\
& \geq \frac{n}{2} \log \left(\frac{d_{L}+\epsilon+\sigma_{Z_{L-1}}^{2}}{d_{L}+\epsilon}\right) \\
& =\min _{\gamma_{L} \in\left[0, d_{L}+\epsilon\right]} \frac{n}{2} \log \left(\frac{\gamma_{L}+\sigma_{Z_{L-1}}^{2}}{\gamma_{L}}\right)
\end{aligned}
$$

where (14) is due to Lemma 8 in Appendix A. In view of Lemma 7 in Appendix A and the fact that $\sigma_{X_{i}^{n}+Z_{i-1}^{n} \mid C_{i}}^{2}=\sigma_{X_{i}^{n} \mid C_{i}}^{2}+$ $\sigma_{Z_{i-1}}^{2} \leq \delta_{i}+\epsilon+\sigma_{Z_{i-1}}^{2}$, we have

$$
\begin{aligned}
& -h\left(X_{i}^{n}+Z_{i-1}^{n} \mid C_{i}\right) \\
& \geq-\frac{n}{2} \log \left(2 \pi e\left(\delta_{i}+\epsilon+\sigma_{Z_{i-1}}^{2}\right)\right), \quad i=2, \ldots, L .
\end{aligned}
$$

Substituting (12), (13), (15), (16) into (10) yields

$$
\begin{aligned}
& \sum_{i=1}^{L} \mu_{i}\left(R_{i}+\epsilon\right) \\
& \geq \min _{\gamma_{i} \in\left[0, d_{i}+\epsilon\right], i=1, \ldots, L} \frac{\mu_{1}}{2} \log \left(\frac{\sigma_{X_{1}}^{2}}{\gamma_{1}}\right) \\
& \quad+\sum_{i=2}^{L} \frac{\mu_{i}}{2} \log \frac{\left(\sigma_{X_{i}}^{2}+\sigma_{Z_{i-1}}^{2}\right)\left(\gamma_{i-1}+\sigma_{\Delta_{i-1}}^{2}\right)\left(\gamma_{i}+\sigma_{Z_{i-1}}^{2}\right)}{\left(\gamma_{i-1}+\sigma_{\Delta_{i-1}}^{2}+\sigma_{Z_{i-1}}^{2}\right)\left(\delta_{i}+\epsilon+\sigma_{Z_{i-1}}^{2}\right) \gamma_{i}} .
\end{aligned}
$$

Replacing $\sigma_{Z_{i}}^{2}$ with $\left(\theta_{i}^{-1}-\sigma_{X_{i+1}}^{-2}\right)^{-1}, i=1, \ldots, L-1$, in (17) gives

$$
\sum_{i=1}^{L} \mu_{i}\left(R_{i}+\epsilon\right) \geq \min _{\gamma_{i} \in\left[0, d_{i}+\epsilon\right], i=1, \ldots . L} \psi\left(\bar{\mu}, \bar{\gamma}, \overline{\delta^{(\epsilon)}}, \bar{\theta}\right)
$$

where $\overline{\delta^{(\epsilon)}}=\left(\delta_{2}+\epsilon, \ldots, \delta_{L}+\epsilon\right)$. Note that there is a one-to-one correspondence between $\sigma_{Z_{i}}^{2} \in(0, \infty)$ and $\theta_{i} \in\left(0, \sigma_{X_{i+1}}^{2}\right)$, $i=1, \ldots, L-1$. Let $\overline{\gamma^{\left(\epsilon_{m}\right)}}$ be a minimizer to

$$
\min _{\gamma_{i} \in\left[0, d_{i}+\epsilon_{m}\right], i=1, \ldots, L} \psi\left(\bar{\mu}, \bar{\gamma}, \overline{\delta^{\left(\epsilon_{m}\right)}}, \bar{\theta}\right)
$$

where $\epsilon_{m}>0$, and $\epsilon_{m}$ tends to zero as $m \rightarrow \infty$. Without loss of generality, we assume that $\overline{\gamma^{\left(\epsilon_{m}\right)}}$ converges to some $\overline{\gamma^{*}}$ as $\underline{m \rightarrow \infty}$ (otherwise, one can take a converging subsequence of $\left.\overline{\gamma^{\left(\epsilon_{m}\right)}}, m \geq 1\right)$. We have

$$
\begin{aligned}
\bar{\mu} \bar{R}^{T} & =\lim _{m \rightarrow \infty} \sum_{i=1}^{L} \mu_{i}\left(R_{i}+\epsilon_{m}\right) \\
& \geq \lim _{m \rightarrow \infty} \psi\left(\bar{\mu}, \overline{\gamma^{\left(\epsilon_{m}\right)}}, \overline{\delta^{\left(\epsilon_{m}\right)}}, \bar{\theta}\right) \\
& =\psi\left(\bar{\mu}, \overline{\gamma^{*}}, \bar{\delta}, \bar{\theta}\right) \\
& \geq \min _{\gamma_{i} \in\left[0, d_{i}\right], i=1, \ldots, L} \psi(\bar{\mu}, \bar{\gamma}, \bar{\delta}, \bar{\theta}) .
\end{aligned}
$$

This completes the proof of (6).

\section{Proof of Theorem 2}

It suffices to prove that

$$
\inf _{\bar{R} \in \mathcal{R}_{P}(\bar{d}, \bar{\delta})} \bar{\mu} \bar{R}^{T} \leq \max _{\theta_{i} \in\left[0, \sigma_{X_{i+1}}^{2}\right], i=1, \ldots, L-1} \psi(\bar{\mu}, \bar{\gamma}, \bar{\delta}, \bar{\theta})
$$

for all $\bar{\gamma}$ with $\gamma_{i} \in\left(0, d_{i}\right), i=1, \ldots, L$.

The maximization problem in (18) can be decomposed into

$$
\begin{aligned}
& \max _{\theta_{i} \in\left[0, \sigma_{X_{i+1}}^{2}\right]} \frac{\left(\sigma_{X_{i+1}}^{2}-\theta_{i}\right) \gamma_{i+1}+\sigma_{X_{i+1}}^{2} \theta_{i}}{\left(\left(\sigma_{X_{i+1}}^{2}-\theta_{i}\right)\left(\gamma_{i}+\sigma_{\Delta_{i}}^{2}\right)+\sigma_{X_{i+1}}^{2} \theta_{i}\right)} \\
& \quad \times \frac{1}{\left(\left(\sigma_{X_{i+1}}^{2}-\theta_{i}\right) \delta_{i+1}+\sigma_{X_{i+1}}^{2} \theta_{i}\right)}, \quad i=1, \ldots, L-1 .
\end{aligned}
$$


The maximizers to (19) are characterized by the following result [7, Lemma 2].

Lemma 1: For $i=1, \ldots, L-1$, define

$$
\begin{aligned}
& \gamma_{i+1}^{\diamond}=\left(\frac{1}{\gamma_{i}+\sigma_{\Delta_{i}}^{2}}+\frac{1}{\delta_{i+1}}-\frac{1}{\sigma_{X_{i+1}}^{2}}\right)^{-1}, \\
& \gamma_{i+1}^{\star}=\gamma_{i}+\sigma_{\Delta_{i}}^{2}+\delta_{i+1}-\sigma_{X_{i+1}}^{2} .
\end{aligned}
$$

1) If $\max \left\{\gamma_{i}+\sigma_{\Delta_{i}}^{2}, \delta_{i+1}\right\}<\sigma_{X_{i+1}}^{2}$, then $\gamma_{i+1}^{\diamond}>\gamma_{i+1}^{\star}$ and the maximizers to (19) are given by

$\theta_{i}= \begin{cases}0, & \gamma_{i+1} \geq \gamma_{i+1}^{\diamond} \\ \sigma_{X_{i+1}}^{2}, & \gamma_{i+1} \leq \gamma_{i+1}^{\star}, \quad i=1, \ldots, L-1 \\ \hat{\theta}_{i}, & \text { otherwise }\end{cases}$

where

$$
\begin{aligned}
\hat{\theta}_{i} \triangleq & \sqrt{\left(\frac{\sigma_{X_{i+1}}^{2}\left(\gamma_{i}+\sigma_{\Delta_{i}}^{2}\right)}{\sigma_{X_{i+1}}^{2}-\gamma_{i}-\sigma_{\Delta_{i}}^{2}}-\frac{\sigma_{X_{i+1}}^{2} \gamma_{i+1}}{\sigma_{X_{i+1}}^{2}-\gamma_{i+1}}\right)} \\
& \times \sqrt{\left(\frac{\sigma_{X_{i+1}}^{2} \delta_{i+1}}{\sigma_{X_{i+1}}^{2}-\delta_{i+1}}-\frac{\sigma_{X_{i+1}}^{2} \gamma_{i+1}}{\sigma_{X_{i+1}}^{2}-\gamma_{i+1}}\right)} \\
& -\frac{\sigma_{X_{i+1}}^{2} \gamma_{i+1}}{\sigma_{X_{i+1}}^{2}-\gamma_{i+1}}
\end{aligned}
$$

is the unique solution to the following equation:

$$
\begin{aligned}
& \left(\frac{\sigma_{X_{i+1}}^{2} \gamma_{i+1}}{\sigma_{X_{i+1}}^{2}-\gamma_{i+1}}+\hat{\theta}_{i}\right)^{-1} \\
& =\left(\frac{\sigma_{X_{i+1}}^{2}\left(\gamma_{i}+\sigma_{\Delta_{i}}^{2}\right)}{\sigma_{X_{i+1}}^{2}-\gamma_{i}-\sigma_{\Delta_{i}}^{2}}+\hat{\theta}_{i}\right)^{-1} \\
& \quad+\left(\frac{\sigma_{X_{i+1}}^{2} \delta_{i+1}}{\sigma_{X_{i+1}}^{2}-\delta_{i+1}}+\hat{\theta}_{i}\right)^{-1}
\end{aligned}
$$

for $\hat{\theta}_{i} \in\left(0, \sigma_{X_{i+1}}^{2}\right), i=1, \ldots, L-1$. The maximizers $\theta_{i}, i=1, \ldots, L-1$, given in (20) are monotonically increasing continuous functions of $\gamma_{i}$ and monotonically decreasing continuous functions of $\gamma_{i+1}$; furthermore, the monotonicity is strict when $\theta_{i} \in\left(0, \sigma_{X_{i+1}}^{2}\right)$.

2) If $\max \left\{\gamma_{i}+\sigma_{\Delta_{i}}^{2}, \delta_{i+1}\right\}=\sigma_{X_{i+1}}^{2}$, then $\gamma_{i+1}^{\diamond}=\gamma_{i+1}^{\star}=$ $\min \left\{\gamma_{i}+\sigma_{\Delta_{i}}^{2}, \delta_{i+1}\right\}$ and the maximizers to (19) are given by

$$
\theta_{i}= \begin{cases}0, & \gamma_{i+1}>\min \left\{\gamma_{i}+\sigma_{\Delta_{i}}^{2}, \delta_{i+1}\right\} \\ \sigma_{X_{i+1}}^{2}, & \gamma_{i+1}<\min \left\{\gamma_{i}+\sigma_{\Delta_{i}}^{2}, \delta_{i+1}\right\} \\ \text { any number in }\left[0, \sigma_{X_{i+1}}^{2}\right], \quad \text { otherwise } & \\ i=1, \ldots, L-1 .\end{cases}
$$

The proof of the following lemma is essentially the same as that of [7, Lemma 3] and thus is omitted.

Lemma 2: There exist $\overline{\gamma^{\prime}} \triangleq\left(\gamma_{1}^{\prime}, \ldots, \gamma_{L}^{\prime}\right)$ and $\overline{\delta^{\prime}} \triangleq\left(\delta_{2}^{\prime}, \ldots, \delta_{L}^{\prime}\right)$ with

$$
\begin{aligned}
& 0<\gamma_{i}^{\prime} \leq \gamma_{i}, \quad i=1, \ldots, L \\
& 0<\delta_{i}^{\prime} \leq \delta_{i}, \quad i=2, \ldots, L \\
& \gamma_{i}^{\prime}+\sigma_{\Delta_{i}}^{2}+\delta_{i+1}^{\prime}-\sigma_{X_{i+1}}^{2} \\
& \leq \gamma_{i+1}^{\prime} \leq\left(\frac{1}{\gamma_{i}^{\prime}+\sigma_{\Delta_{i}}^{2}}+\frac{1}{\delta_{i+1}^{\prime}}-\frac{1}{\sigma_{X_{i+1}}^{2}}\right)^{-1}, \\
& \quad i=1, \ldots, L-1
\end{aligned}
$$

such that

$$
\begin{aligned}
& \max _{\theta_{i} \in\left[0, \sigma_{X_{i+1}}^{2}\right], i=1, \ldots, L-1} \psi\left(\bar{\mu}, \overline{\gamma^{\prime}}, \overline{\delta^{\prime}}, \bar{\theta}\right) \\
& \leq \max _{\theta_{i} \in\left[0, \sigma_{X_{i+1}}^{2}\right], i=1, \ldots, L-1} \psi(\bar{\mu}, \bar{\gamma}, \bar{\delta}, \bar{\theta}) .
\end{aligned}
$$

The following lemma provides an inner bound of $\mathcal{R}_{P}(\bar{d}, \bar{\delta})$. A sketch of the proof is given in Appendix B.

Lemma 3: Let $\left(U_{1}, \ldots, U_{L}\right)$ be jointly Gaussian with $\left(X_{1}, \ldots, X_{L}\right)$ such that

1) $\left(X_{2}, \ldots, X_{L}\right) \leftrightarrow X_{1} \leftrightarrow U_{1}$ form a Markov chain, and $\left(X_{j}\right)_{j \neq i} \leftrightarrow\left(X_{i}, U_{1}, \ldots, U_{i-1}\right) \leftrightarrow U_{i}$ form a Markov chain, $i=1, \ldots, L$,

2) $\sigma_{X_{i}{ }^{\prime} U_{1}, \ldots, U_{i}}^{2} \leq d_{i}, i=1, \ldots, L$, and $\sigma_{X_{i} \mid U_{i}}^{2} \leq \delta_{i}, i=$ Then, $\bar{R} \in \mathcal{R}_{P}(\bar{d}, \bar{\delta})$ for any $\bar{R}$ satisfying

$$
\begin{aligned}
& R_{1} \geq I\left(X_{1} ; U_{1}\right), \\
& R_{i} \geq I\left(X_{i}, U_{1}, \ldots, U_{i-1} ; U_{i}\right), \quad i=2, \ldots, L .
\end{aligned}
$$

For the purpose of proving Theorem 2, it suffices to construct $\left(U_{1}, \ldots, U_{L}\right)$ satisfying the conditions in Lemma 3 such that

$$
\begin{aligned}
& \mu_{1} I\left(X_{1} ; U_{1}\right)+\sum_{i=2}^{L} \mu_{i} I\left(X_{i}, U_{1}, \ldots, U_{i-1} ; U_{i}\right) \\
& =\operatorname{\theta ax}_{\theta_{i} \in\left[0, \sigma_{X_{i+1}}^{2}\right], i=1, \ldots, L-1} \psi\left(\bar{\mu}, \overline{\gamma^{\prime}}, \overline{\delta^{\prime}}, \bar{\theta}\right)
\end{aligned}
$$

where $\overline{\gamma^{\prime}}$ and $\overline{\delta^{\prime}}$ are specified in Lemma 2. Define

$$
\begin{aligned}
& \xi_{i}= \begin{cases}0, & \max \left\{\gamma_{i}^{\prime}+\sigma_{\Delta_{i}}^{2}, \delta_{i+1}^{\prime}\right\}=\sigma_{X_{i+1}}^{2} \\
\frac{\sigma_{X_{i+1}}^{4}\left(\sigma_{X_{i+1}}^{2}+\varsigma_{i}-\sqrt{\left(\tau_{i}-\varsigma_{i}\right)\left(\lambda_{i}-\varsigma_{i}\right)}\right)}{\left(\sigma_{X_{i+1}}^{2}+\tau_{i}\right)\left(\sigma_{X_{i+1}}^{2}+\lambda_{i}\right)}, & \text { otherwise }\end{cases} \\
& \left\{\begin{array}{cc}
0, & i=1, \ldots, L-1 \\
& \gamma_{i}^{\prime}+\sigma_{\Delta_{i}}^{2}=\sigma_{X_{i+1}}^{2}
\end{array}\right. \\
& a_{i}= \begin{cases}\frac{\left(\sigma_{X_{i+1}}^{2}+\tau_{i}\right) \sqrt{\lambda_{i}-\varsigma_{i}}}{\left(\sigma_{X_{i+1}}^{2}+\varsigma_{i}\right)\left(\sqrt{\tau_{i}-\varsigma_{i}}+\sqrt{\lambda_{i}-\varsigma_{i}}\right)}, & \text { otherwise }\end{cases} \\
& i=1, \ldots, L-1 \\
& \begin{cases}0, & \delta_{i+1}^{\prime}=\sigma_{X_{i+1}}^{2}\end{cases} \\
& b_{i}= \begin{cases}\frac{\left(\sigma_{X_{i+1}}^{2}+\lambda_{i}\right) \sqrt{\tau_{i}-\varsigma_{i}}}{\left(\sigma_{X_{i+1}}^{2}+\varsigma_{i}\right)\left(\sqrt{\tau_{i}-\varsigma_{i}}+\sqrt{\lambda_{i}-\varsigma_{i}}\right)}, & \text { otherwise }\end{cases} \\
& \theta_{i}^{\prime}=\left\{\begin{array}{c}
\text { any number in }\left[0, \sigma_{X_{i+1}}^{2}\right], \\
\max \left\{\gamma_{i}^{\prime}+\sigma_{\Delta_{i}}^{2}, \delta_{i+1}^{\prime}\right\}=\sigma_{X_{i+1}}^{2} \\
\sqrt{\left(\tau_{i}-\varsigma_{i}\right)\left(\lambda_{i}-\varsigma_{i}\right)}-\varsigma_{i}, \quad \text { otherwise }
\end{array}\right. \\
& i=1, \ldots, L-1
\end{aligned}
$$

where

$$
\begin{aligned}
\tau_{i} & =\frac{\sigma_{X_{i+1}}^{2}\left(\gamma_{i}^{\prime}+\sigma_{\Delta_{i}}^{2}\right)}{\sigma_{X_{i+1}}^{2}-\gamma_{i}^{\prime}-\sigma_{\Delta_{i}}^{2}}, \quad i=1, \ldots, L-1 \\
\lambda_{i} & =\frac{\sigma_{X_{i+1}}^{2} \delta_{i+1}^{\prime}}{\sigma_{X_{i+1}}^{2}-\delta_{i+1}^{\prime}}, \quad i=1, \ldots, L-1 \\
\varsigma_{i} & =\frac{\sigma_{X_{i+1}}^{2} \gamma_{i+1}^{\prime}}{\sigma_{X_{i+1}}^{2}-\gamma_{i+1}^{\prime}}, \quad i=1, \ldots, L-1 .
\end{aligned}
$$


We assume $\mathbb{E}\left[U_{i}\right]=0, i=1, \ldots, L$. Let $U_{1}$ be jointly Gaussian with $\left(X_{1}, \ldots, X_{L}\right)$ such that

$$
\mathbb{E}\left[U_{1}^{2}\right]=\mathbb{E}\left[X_{1} U_{1}\right]=\sigma_{X_{1}}^{2}-\gamma_{1}^{\prime}
$$

and $\left(X_{2}, \ldots, X_{L}\right) \leftrightarrow X_{1} \leftrightarrow U_{1}$ form a Markov chain. Now let $U_{i}$ be jointly Gaussian with $\left(X_{1}, \ldots, X_{L}, U_{1}, \ldots, U_{i-1}\right)$ such that

$$
\begin{aligned}
& \mathbb{E}\left[U_{i}^{2}\right]=\mathbb{E}\left[X_{i} U_{i}\right]=\sigma_{X_{i}}^{2}-\delta_{i}^{\prime} \\
& \mathbb{E}\left[\tilde{U}_{i-1} U_{i}\right]=\xi_{i-1}
\end{aligned}
$$

and $\left(\left(X_{j}\right)_{j \neq i}, U_{1}, \ldots, U_{i-1}\right) \leftrightarrow\left(X_{i}, \tilde{U}_{i-1}\right) \leftrightarrow U_{i}$ form a Markov chain, $i=2, \ldots, L$, where

$$
\begin{aligned}
& \tilde{U}_{1}=U_{1} \\
& \tilde{U}_{i}=a_{i-1} \tilde{U}_{i-1}+b_{i-1} U_{i}, \quad i=2, \ldots, L .
\end{aligned}
$$

It is clear that the covariance matrix of $\left(X_{1}, U_{1}\right)$ is positive semidefinite; moreover, one can readily verify that the covariance matrix of $\left(X_{i}, \tilde{U}_{i-1}, U_{i}\right)$ is positive semidefinite, $i=2, \ldots, L$. As a consequence, the joint distribution of $\left(X_{1}, \ldots, X_{L}\right)$ and the constructed $\left(U_{1}, \ldots, U_{L}\right)$ (as well as the induced $\left.\left(\tilde{U}_{1}, \ldots, \tilde{U}_{L}\right)\right)$ is well defined. It can be verified that

$$
\begin{aligned}
& \tilde{U}_{1}=\mathbb{E}\left[X_{1} \mid U_{1}\right] \\
& \tilde{U}_{i}=\mathbb{E}\left[X_{i} \mid U_{1}, \ldots, U_{i}\right]=\mathbb{E}\left[X_{i} \mid \tilde{U}_{i-1}, U_{i}\right], \quad i=2, \ldots, L \\
& \mathbb{E}\left[\left(X_{i}-\tilde{U}_{i}\right)^{2}\right]=\gamma_{i}^{\prime}, \quad i=1, \ldots, L \\
& \mathbb{E}\left[\left(X_{i}-U_{i}\right)^{2}\right]=\delta_{i}^{\prime}, \quad i=2, \ldots, L .
\end{aligned}
$$

Therefore, the constructed $\left(U_{1}, \ldots, U_{L}\right)$ satisfies the conditions in Lemma 3. Note that

$$
I\left(X_{1} ; U_{1}\right)=\frac{1}{2} \log \left(\frac{\sigma_{X_{1}}^{2}}{\gamma_{1}^{\prime}}\right) .
$$

Moreover, we have

$$
\begin{aligned}
& I\left(X_{i}, U_{1}, \ldots, U_{i-1} ; U_{i}\right) \\
& =I\left(X_{i}, \tilde{U}_{i-1} ; U_{i}\right) \\
& =\frac{1}{2} \log \left(\frac{\sigma_{X_{i}}^{4}\left(\gamma_{i-1}^{\prime}+\sigma_{\Delta_{i-1}}^{2}\right)}{\left(\left(\sigma_{X_{i}}^{2}-\theta_{i-1}^{\prime}\right)\left(\gamma_{i-1}+\sigma_{\Delta_{i-1}}^{2}\right)+\sigma_{X_{i}}^{2} \theta_{i-1}^{\prime}\right)}\right. \\
& \left.\quad \times \frac{\left(\left(\sigma_{X_{i}}^{2}-\theta_{i-1}^{\prime}\right) \gamma_{i}^{\prime}+\sigma_{X_{i}}^{2} \theta_{i-1}^{\prime}\right)}{\left(\left(\sigma_{X_{i}}^{2}-\theta_{i-1}^{\prime}\right) \delta_{i}^{\prime}+\sigma_{X_{i}}^{2} \theta_{i-1}^{\prime}\right) \gamma_{i}^{\prime}}\right), \quad i=2, \ldots, L
\end{aligned}
$$

where (27) is due to the fact that $\left(X_{i}, U_{1}, \ldots, U_{i-1}\right) \leftrightarrow$ $\left(X_{i}, \tilde{U}_{i}\right) \leftrightarrow U_{i}$ form a Markov chain and that $\tilde{U}_{i}$ is a (linear) function of $\left(U_{1}, \ldots, U_{i-1}\right)$, and (28) is by direct evaluation (see (26) for the definition of $\theta_{i}^{\prime}, i=1, \ldots, L-1$ ). Now one can readily prove (22) by invoking Lemma 1 . This completes the proof of Theorem 2 .

\section{Proof of Theorem 3}

Without loss of generality, we assume $\mu_{1} \geq \cdots \geq \mu_{L}>0$ throughout this proof. Note that the minimization problem

$$
\min _{\gamma_{i} \in\left[0, d_{i}\right], i=1, \ldots, L} \psi(\bar{\mu}, \bar{\gamma}, \bar{\delta}, \bar{\theta})
$$

can be decomposed into

$$
\begin{aligned}
& \min _{\gamma_{1} \in\left[0, d_{1}\right]} \frac{\mu_{1}}{2} \log \left(\frac{\sigma_{X_{1}}^{2}}{\gamma_{1}}\right) \\
&+ \frac{\mu_{2}}{2} \log \left(\frac{\gamma_{1}+\sigma_{\Delta_{1}}^{2}}{\left(\sigma_{X_{2}}^{2}-\theta_{1}\right)\left(\gamma_{1}+\sigma_{\Delta_{1}}^{2}\right)+\sigma_{X_{2}}^{2} \theta_{1}}\right) \\
& \min _{\gamma_{i} \in\left[0, d_{i}\right]} \frac{\mu_{i}}{2} \log \left(\frac{\left(\sigma_{X_{i}}^{2}-\theta_{i-1}\right) \gamma_{i}+\sigma_{X_{i}}^{2} \theta_{i-1}}{\gamma_{i}}\right) \\
&+\frac{\mu_{i+1}}{2} \log \left(\frac{\gamma_{i}+\sigma_{\Delta_{i}}^{2}}{\left(\sigma_{X_{i+1}}^{2}-\theta_{i}\right)\left(\gamma_{i}+\sigma_{\Delta_{i}}^{2}\right)+\sigma_{X_{i+1}}^{2} \theta_{i}}\right), \\
& i=2, \ldots, L-1 \\
& \min _{\gamma_{L} \in\left[0, d_{L}\right]} \frac{\mu_{L}}{2} \log \left(\frac{\left(\sigma_{X_{L}}^{2}-\theta_{L-1}\right) \gamma_{L}+\sigma_{X_{L}}^{2} \theta_{L-1}}{\gamma_{L}}\right) .
\end{aligned}
$$

The minimizers to (29)-(31) are characterized by the following lemmas. The proofs of Lemmas 4 and 6 are straightforward and thus omitted. The proof of Lemma 5 is given in Appendix C.

Lemma 4: The minimizer to (29) is given by $\gamma_{1}=d_{1}$.

Lemma 5: For $i=2, \ldots, L-1$, define

$$
\begin{aligned}
\tilde{a}_{i}= & \mu_{i+1}\left(\sigma_{X_{i}}^{2}-\theta_{i-1}\right) \sigma_{X_{i+1}}^{2} \theta_{i} \\
& -\mu_{i}\left(\sigma_{X_{i+1}}^{2}-\theta_{i}\right) \sigma_{X_{i}}^{2} \theta_{i-1} \\
\tilde{b}_{i}= & \left(\mu_{i+1}-\mu_{i}\right) \sigma_{X_{i}}^{2} \sigma_{X_{i+1}}^{2} \theta_{i-1} \theta_{i} \\
& -2 \mu_{i} \sigma_{X_{i}}^{2} \theta_{i-1} \sigma_{\Delta_{i}}^{2}\left(\sigma_{X_{i+1}}^{2}-\theta_{i}\right) \\
\tilde{c}_{i}= & -\mu_{i} \sigma_{X_{i}}^{2} \theta_{i-1} \sigma_{\Delta_{i}}^{2}\left(\sigma_{X_{i+1}}^{2} \sigma_{\Delta_{i}}^{2}+\sigma_{X_{i}}^{2} \theta_{i}\right) .
\end{aligned}
$$

The minimizers to (30) are given by

$$
\gamma_{i}= \begin{cases}\min \left\{\hat{\gamma}_{i}, d_{i}\right\}, & \tilde{a}_{i}>0 \\ d_{i}, & \tilde{a}_{i} \leq 0, \theta_{i-1} \in\left(0, \sigma_{X_{i}}^{2}\right] \\ \text { any number in }\left[0, d_{i}\right], & \theta_{i-1}=\theta_{i}=0 \\ & i=2, \ldots, L-1\end{cases}
$$

where

$$
\hat{\gamma}_{i}=\frac{-\tilde{b}_{i}+\sqrt{\tilde{b}_{i}^{2}-4 \tilde{a}_{i} \tilde{c}_{i}}}{2 \tilde{a}_{i}}, \quad i=2, \ldots, L-1 .
$$

Lemma 6: The minimizer to (31) is given by

$$
\gamma_{L}= \begin{cases}d_{L}, & \theta_{L-1} \in\left(0, \sigma_{X_{L}}^{2}\right] \\ \text { any number in }\left[0, d_{L}\right], & \theta_{L-1}=0 .\end{cases}
$$

Now we proceed to prove Theorem 3 . The key step is to show the existence of a saddle point $\left(\overline{\gamma^{*}}, \overline{\theta^{*}}\right)$ with the property that

$$
\begin{aligned}
& \psi\left(\bar{\mu}, \overline{\gamma^{*}}, \bar{\delta}, \overline{\theta^{*}}\right)=\min _{\gamma_{i} \in\left[0, d_{i}\right], i=1, \ldots, L} \psi\left(\bar{\mu}, \bar{\gamma}, \bar{\delta}, \overline{\theta^{*}}\right) \\
& \psi\left(\bar{\mu}, \overline{\gamma^{*}}, \bar{\delta}, \overline{\theta^{*}}\right)=\max _{\theta_{i} \in\left[0, \sigma_{X_{i+1}}^{2} \max _{i=1, \ldots, L-1}\right.} \psi\left(\bar{\mu}, \overline{\gamma^{*}}, \bar{\delta}, \bar{\theta}\right) .
\end{aligned}
$$

First consider the case where $d_{i}<\sigma_{X_{i}}^{2}, i=1, \ldots, L$, and $\delta_{i}<\sigma_{X_{i}}^{2}, i=2, \ldots, L$. Let $\gamma_{1}^{*}=d_{1}$ and $\gamma_{L}^{*}=d_{L}$. Define

$$
\begin{aligned}
& \omega\left(\gamma_{2}, \ldots, \gamma_{L-1}\right) \\
& =\left.\max _{\theta_{i} \in\left[0, \sigma_{X_{i+1}}^{2}\right], i=1, \ldots, L-1} \psi(\bar{\mu}, \bar{\gamma}, \bar{\delta}, \bar{\theta})\right|_{\gamma_{1}=\gamma_{1}^{*}, \gamma_{L}=\gamma_{L}^{*}} .
\end{aligned}
$$

Note that $\omega\left(\gamma_{2}, \ldots, \gamma_{L-1}\right)$ is a continuous function of $\left(\gamma_{2}, \ldots, \gamma_{L-1}\right)$ for $\gamma_{i} \in\left(0, \sigma_{X_{i}}^{2}\right], i=2, \ldots, L-1$, and 
$\omega\left(\gamma_{2}, \ldots, \gamma_{L-1}\right)=\infty$ if $\gamma_{i}=0$ for some $i$; moreover, it is clear from the proof of Theorem 2 that

$$
\omega\left(\gamma_{2}, \ldots, \gamma_{L-1}\right) \geq \frac{\mu_{i}}{2} \log \left(\frac{\sigma_{X_{i}}^{2}}{\gamma_{i}}\right), \quad i=2, \ldots, L-1 .
$$

Therefore, the minimum of $\omega\left(\gamma_{2}, \ldots, \gamma_{L-1}\right)$ over $\left(\gamma_{2}, \ldots, \gamma_{L-1}\right)$ with $\gamma_{i} \in\left[0, \sigma_{X_{i}}^{2}\right], i=2, \ldots, L-1$, is achieved at some $\left(\gamma_{2}^{*}, \ldots, \gamma_{L-1}^{*}\right)$ satisfying $\gamma_{i}^{*} \in\left(0, \sigma_{X_{i}}^{2}\right]$, $i=2, \ldots, L-1$. Let $\overline{\theta^{*}} \triangleq\left(\theta_{1}^{*}, \ldots, \theta_{L}^{*}\right)$ be a maximizer to

$$
\max _{\theta_{i} \in\left[0, \sigma_{X_{i}}^{2}\right], i=1, \ldots, L-1} \psi\left(\bar{\mu}, \overline{\gamma^{*}}, \bar{\delta}, \bar{\theta}\right)
$$

where $\overline{\gamma^{*}}=\left(\gamma_{1}^{*}, \ldots, \gamma_{L}^{*}\right)$. We shall prove that $\left(\overline{\gamma^{*}}, \overline{\theta^{*}}\right)$ satisfies (38) (note that (39) is automatically satisfied).

In view of Lemmas 4 and 6 , and our choice of $\left(\gamma_{1}^{*}, \gamma_{L}^{*}\right)$, we just need to show that $\left(\gamma_{2}^{*}, \ldots, \gamma_{L-1}^{*}\right)$ satisfies the optimality condition (35) (with $\theta_{i}=\theta_{i}^{*}, i=1, \ldots, L-1$ ) in Lemma 5. Let us assume that (35) is violated by $\gamma_{i^{*}}^{*}$ for some $i^{*}$. Note that $\theta_{i^{*}-1}^{*}$ and $\theta_{i^{*}}^{*}$ are determined by $\gamma_{i^{*}}^{*}$ according to (20). To stress this dependence, we denote $\theta_{i^{*}-1}^{*}$ and $\theta_{i^{*}}^{*}$ by $\theta_{i^{*}-1}^{*}\left(\gamma_{i^{*}}^{*}\right)$ and $\theta_{i^{*}}^{*}\left(\gamma_{i^{*}}^{*}\right)$, respectively. It is clear that at least one of $\theta_{i^{*}-1}^{*}\left(\gamma_{i^{*}}^{*}\right)$ and $\theta_{i^{*}}^{*}\left(\gamma_{i^{*}}^{*}\right)$ is not zero since otherwise (35) is satisfied by $\gamma_{i^{*}}^{*}$. Now, let $\tilde{\gamma}_{i^{*}}\left(\gamma_{i^{*}}^{*}\right)$ be the minimizer determined by $\theta_{i^{*}-1}^{*}\left(\gamma_{i^{*}}^{*}\right)$ and $\theta_{i^{*}}^{*}\left(\gamma_{i^{*}}^{*}\right)$ according to (35). We shall move $\gamma_{i^{*}}^{*}$ toward $\tilde{\gamma}_{i^{*}}\left(\gamma_{i^{*}}^{*}\right)$ (and change $\theta_{i^{*}-1}^{*}\left(\gamma_{i^{*}}^{*}\right), \theta_{i^{*}}^{*}\left(\gamma_{i^{*}}^{*}\right)$, and $\tilde{\gamma}_{i^{*}}\left(\gamma_{i^{*}}^{*}\right)$ correspondingly) while keeping $\gamma_{i}^{*}\left(i \neq i^{*}\right)$ and $\theta_{i}^{*}\left(i \neq i^{*}-1\right.$ and $\left.i \neq i^{*}\right)$ fixed. It is shown in Appendix D that $\tilde{\gamma}_{i^{*}}\left(\gamma_{i^{*}}^{*}\right)$ varies continuously with $\gamma_{i^{*}}^{*}$ if at least one of $\theta_{i^{*}-1}^{*}\left(\gamma_{i^{*}}^{*}\right)$ and $\theta_{i^{*}}^{*}\left(\gamma_{i^{*}}^{*}\right)$ is not zero. As a consequence, we can keep moving $\gamma_{i^{*}}^{*}$ until $\gamma_{i^{*}}^{*}=\tilde{\gamma}_{i^{*}}^{*}$ at which one of the following cases happens:

1) $\tilde{\gamma}_{i^{*}}^{*}=\tilde{\gamma}_{i^{*}}\left(\tilde{\gamma}_{i^{*}}^{*}\right)$;

2) $\theta_{i^{*}-1}^{*}\left(\tilde{\gamma}_{i^{*}}^{*}\right)=\theta_{i^{*}}^{*}\left(\tilde{\gamma}_{i^{*}}^{*}\right)=0$.

Clearly, $\tilde{\gamma}_{i^{*}}^{*}$ satisfies (35) with $\theta_{i^{*}-1}=\theta_{i^{*}-1}^{*}\left(\tilde{\gamma}_{i^{*}}^{*}\right)$ and $\theta_{i^{*}}=$ $\theta_{i^{*}}^{*}\left(\tilde{\gamma}_{i^{*}}^{*}\right)$. Define $\overline{\gamma^{\circ}}=\left(\gamma_{1}^{\circ}, \ldots, \gamma_{L}^{\circ}\right)$ with $\gamma_{i^{*}}^{\circ}=\tilde{\gamma}_{i^{*}}^{*}$ and $\gamma_{i}^{\circ}=$ $\gamma_{i}^{*}$ for $i \neq i^{*}$. Moreover, define $\bar{\theta}^{\circ}=\left(\theta_{1}^{\circ}, \ldots, \theta_{L}^{\circ}\right)$ with $\theta_{i^{*}-1}^{\circ}=$ $\theta_{i^{*}-1}^{*}\left(\tilde{\gamma}_{i^{*}}^{*}\right), \theta_{i^{*}}^{\circ}=\theta_{i^{*}}^{*}\left(\tilde{\gamma}_{i^{*}}^{*}\right)$, and $\theta_{i}^{\circ}=\theta_{i}^{*}$ for $i \neq i^{*}-1$ and $i \neq i^{*}$. If $\overline{\theta^{\circ}} \neq \overline{\theta^{*}}$, then

$$
\begin{aligned}
& \psi\left(\bar{\mu}, \overline{\gamma^{*}}, \bar{\delta}, \overline{\theta^{*}}\right) \\
& >\psi\left(\bar{\mu}, \overline{\gamma^{*}}, \bar{\delta}, \overline{\theta^{\circ}}\right) \\
& \geq \psi\left(\bar{\mu}, \overline{\gamma^{\circ}}, \bar{\delta}, \overline{\theta^{\circ}}\right) \\
& =\max _{\theta_{i} \in\left[0, \sigma_{X_{i+1}}^{2}\right], i=1, \ldots, L-1} \psi\left(\bar{\mu}, \overline{\gamma^{\circ}}, \bar{\delta}, \bar{\theta}\right) .
\end{aligned}
$$

If $\overline{\theta^{\circ}}=\overline{\theta^{*}}$, then

$$
\begin{aligned}
& \psi\left(\bar{\mu}, \overline{\gamma^{*}}, \bar{\delta}, \overline{\theta^{*}}\right) \\
& >\psi\left(\bar{\mu}, \overline{\gamma^{\circ}}, \bar{\delta}, \overline{\theta^{*}}\right) \\
& =\operatorname{\theta ix}_{i} \in\left[0, \sigma_{X_{i+1}}^{2} \max _{i=1, \ldots, L-1} \psi\left(\bar{\mu}, \overline{\gamma^{\circ}}, \bar{\delta}, \bar{\theta}\right) .\right.
\end{aligned}
$$

Note that both (40) and (41) contradict with the fact that $\left(\gamma_{2}^{*}, \ldots, \gamma_{L-1}^{*}\right)$ achieves the minimum of $\omega\left(\gamma_{2}, \ldots, \gamma_{L-1}\right)$ over $\left(\gamma_{2}, \ldots, \gamma_{L-1}\right)$ with $\gamma_{i} \in\left[0, \sigma_{X_{i}}^{2}\right], i=2, \ldots, L-1$. Therefore, $\left(\overline{\gamma^{*}}, \overline{\theta^{*}}\right)$ indeed satisfies (38) and thus is a saddle point.
Now consider the general case where $d_{i} \in\left(0, \sigma_{X_{i}}^{2}\right], i=$ $1, \ldots, L$, and $\delta_{i} \in\left(0, \sigma_{X_{i}}^{2}\right], i=2, \ldots, L$. The preceding argument shows the existence of $\left(\overline{\gamma^{(k)}}, \overline{\theta^{(k)}}\right)$ such that

$$
\begin{aligned}
\psi\left(\bar{\mu}, \overline{\gamma^{(k)}}, \bar{\delta}, \overline{\theta^{(k)}}\right) & =\min _{\gamma_{i} \in\left[0, \rho_{k} d_{i}\right], i=1, \ldots, L} \psi\left(\bar{\mu}, \bar{\gamma}, \rho_{k} \bar{\delta}, \overline{\theta^{(k)}}\right) \\
\psi\left(\bar{\mu}, \overline{\gamma^{(k)}}, \bar{\delta}, \overline{\theta^{(k)}}\right) & =\max _{\theta_{i} \in\left[0, \sigma_{X_{i+1}}^{2}\right], i=1, \ldots, L-1} \psi\left(\bar{\mu}, \overline{\gamma^{(k)}}, \rho_{k} \bar{\delta}, \bar{\theta}\right)
\end{aligned}
$$

where $\rho_{k} \in(0,1)$, and $\rho_{k}$ tends to one as $k \rightarrow \infty$. By taking a converging subsequence of $\left(\overline{\gamma^{(k)}}, \overline{\theta^{(k)}}\right), k \geq 1$, with its limit denoted by $\left(\overline{\gamma^{*}}, \overline{\theta^{*}}\right)$, one can readily verify that $\left(\overline{\gamma^{*}}, \overline{\theta^{*}}\right)$ satisfies (38) and (39)

Let $\left(\overline{\gamma^{*}}, \overline{\theta^{*}}\right)$ be an arbitrary saddle point satisfying (38) and (39). It can be shown ${ }^{2}$ that

S1) $\gamma_{i}^{*}>0, i=1, \ldots, L$;

S2) there exists some $i^{*}$ such that $\theta_{i}^{*}>0$ for $i<i^{*}$ and $\theta_{i}^{*}=0$ for $i \geq i^{*}$ (we set $i^{*}=1$ if all the entries of $\overline{\theta^{*}}$ are zero, and set $i^{*}=L$ if all the entries of $\overline{\theta^{*}}$ are positive).

To complete the proof of Theorem 3 , it suffices to show that

$$
\begin{aligned}
& \kappa_{u}(\bar{\mu}, \bar{d}, \bar{\delta}) \leq \psi\left(\bar{\mu}, \overline{\gamma^{*}}, \bar{\delta}, \overline{\theta^{*}}\right) \\
& \kappa_{l}(\bar{\mu}, \bar{d}, \bar{\delta}) \geq \psi\left(\bar{\mu}, \overline{\gamma^{*}}, \bar{\delta}, \overline{\theta^{*}}\right) .
\end{aligned}
$$

Clearly

$$
\kappa_{u}(\bar{\mu}, \bar{d}, \bar{\delta}) \leq \psi\left(\bar{\mu}, \overline{\gamma^{(m)}}, \bar{\delta}, \overline{\theta^{(m)}}\right)
$$

where $\overline{\gamma^{(m)}} \triangleq\left(\gamma_{1}^{(m)}, \ldots, \gamma_{L}^{(m)}\right) \quad\left(\right.$ with $\gamma_{i}^{(m)} \in\left(0, d_{i}\right)$, $i=1, \ldots, L)$ tends to $\overline{\gamma^{*}}$ as $m \rightarrow \infty$, and $\overline{\theta^{(m)}}$ is a maximizer to

$$
\max _{\theta_{i} \in\left[0, \sigma_{X_{i+1}}^{2}\right], i=1, \ldots, L-1} \psi\left(\bar{\mu}, \overline{\gamma^{(m)}}, \bar{\delta}, \bar{\theta}\right)
$$

Without loss of generality, we assume that $\overline{\theta^{(m)}}$ converges to some $\overline{\theta^{\circ}}$ as $m \rightarrow \infty$ (otherwise one can take a converging subsequence of $\left.\overline{\theta^{(m)}}, m \geq 1\right)$. Note that

$$
\lim _{m \rightarrow \infty} \psi\left(\bar{\mu}, \overline{\gamma^{(m)}}, \bar{\delta}, \overline{\theta^{(m)}}\right)=\psi\left(\bar{\mu}, \overline{\gamma^{*}}, \bar{\delta}, \overline{\theta^{\circ}}\right) \leq \psi\left(\bar{\mu}, \overline{\gamma^{*}}, \bar{\delta}, \overline{\theta^{*}}\right)
$$

where the first equality is due to S1) and the fact that $\psi(\bar{\mu}, \bar{\gamma}, \bar{\delta}, \bar{\theta})$, as a function of $(\bar{\gamma}, \bar{\theta})$, is continuous at $(\bar{\gamma}, \bar{\theta})$ if $\gamma_{i}>0$ for all $i$. Combining (44) and (45) proves (42). Now construct $\overline{\theta^{(n)}} \triangleq\left(\theta_{1}^{(n)}, \ldots, \theta_{L}^{(n)}\right)$ with $\theta_{i}^{(n)} \in\left(0, \sigma_{X_{i+1}}^{2}\right)$, $i=1, \ldots, L-1$, such that $\overline{\theta^{(n)}}$ converges to $\overline{\theta^{*}}$ as $n \rightarrow \infty$, and

$$
\begin{gathered}
\mu_{i+1}\left(\sigma_{X_{i}}^{2}-\theta_{i-1}^{(n)}\right) \sigma_{X_{i+1}}^{2} \theta_{i}^{(n)}-\mu_{i}\left(\sigma_{X_{i+1}}^{2}-\theta_{i}^{(n)}\right) \sigma_{X_{i}}^{2} \theta_{i-1}^{(n)} \leq 0, \\
i=i^{*}+1, \ldots, L-1
\end{gathered}
$$

where (46) is void if $i^{*} \geq L-1$. Let $\overline{\gamma^{(n)}}$ be the minimizer to

$$
\min _{\gamma_{i} \in\left[0, d_{i}\right], i=1, \ldots, L} \psi\left(\bar{\mu}, \bar{\gamma}, \bar{\delta}, \overline{\theta^{(n)}}\right) .
$$

${ }^{2}$ Note that we must have $\gamma_{1}^{*}=d_{1}$. If $\gamma_{i}^{*}=0$ for some $i \geq 2$, then it follows by (20) and (21) that $\theta_{i-1}^{*}>0$; on the other hand, according to (35) and (37), we must have $\theta_{i-1}^{*}=0$, which leads to a contradiction. In view of (35) and the fact that $\gamma_{i}^{*}>0$, we must have $\theta_{i}^{*}=0$ if $\theta_{i-1}^{*}=0$. One can verify that $\psi(\bar{\mu}, \bar{\gamma}, \bar{\delta}, \bar{\theta})$, as a function of $(\bar{\gamma}, \bar{\theta})$, is continuous at $(\bar{\gamma}, \bar{\theta})$ if $\gamma_{i}>0$ for all $i$, but not necessarily so if $\gamma_{i}=0$ for some $i$. It will be seen that S1) and S2) allow us to circumvent such points of discontinuity. 
It is easy to verify that $\overline{\gamma^{(n)}}$ converges to $\overline{\gamma^{\circ}} \triangleq\left(\gamma_{1}^{\circ}, \ldots, \gamma_{L}^{\circ}\right)$ as $n \rightarrow \infty$, where $\gamma_{i}^{\circ}=\gamma^{*}$ for $i<i^{*}$ and $\gamma_{i}^{\circ}=d_{i}$ for $i \geq i^{*}$. Clearly

$$
\begin{aligned}
\kappa_{l}(\bar{\mu}, \bar{d}, \bar{\delta}) & \geq \lim _{n \rightarrow \infty} \psi\left(\bar{\mu}, \overline{\gamma^{(n)}}, \bar{\delta}, \overline{\theta^{(n)}}\right) \\
& =\psi\left(\bar{\mu}, \overline{\gamma^{\circ}}, \bar{\delta}, \overline{\theta^{*}}\right) \\
& \geq \psi\left(\bar{\mu}, \overline{\gamma^{*}}, \bar{\delta}, \overline{\theta^{*}}\right)
\end{aligned}
$$

which proves (43).

\section{MinimAX THEOREM}

We shall show that our main results in Section II can be viewed as a manifestation of a certain information-theoretic minimax theorem. It will be seen that this minimax theorem can be used to explain why there is no loss of optimality in choosing the auxiliary random vectors $Z_{1}^{n}, \ldots, Z_{L-1}^{n}$ to be Gaussian in the proof of Theorem 1.

Let $X_{1}^{n}, \ldots, X_{L}^{n}$ be defined as in Section II. Define

$$
\begin{array}{rl}
\Phi=\mu_{1} & I\left(X_{1}^{n} ; W_{1}\right)+\sum_{i=2}^{L} \mu_{i}\left(I\left(V_{i-1} ; W_{i}\right)\right. \\
& \left.+I\left(X_{i}^{n} ; W_{i} \mid W_{1}, \ldots, W_{i-1}, V_{i-1}\right)\right)
\end{array}
$$

where $\mu_{1} \geq \cdots \geq \mu_{L} \geq 0$. We assume that $V_{i} \leftrightarrow X_{i+1}^{n} \leftrightarrow$ $\left(W_{1}, \ldots, W_{i+1}\right)$ form a Markov chain, $i=1, \ldots, L-1$. As a consequence, in order to determine $\Phi$, it suffices to specify the conditional distribution of $V_{i}$ given $X_{i+1}^{n}, i=1, \ldots, L-1$, as well as the conditional distribution of $\left(W_{1}, \ldots, W_{L}\right)$ given $\left(X_{1}^{n}, \ldots, X_{L}^{n}\right)$. Let $\mathcal{P}$ denote the set of conditional distributions $\left(p_{V_{1} \mid X_{2}^{n}}, \ldots, p_{V_{L-1} \mid X_{L}^{n}}\right)$. Moreover, let $\mathcal{Q}$ denote the set of conditional distributions of $\left(W_{1}, \ldots, W_{L}\right)$ given $\left(X_{1}^{n}, \ldots, X_{L}^{n}\right)$ such that $\sigma_{X_{i}^{n} \mid W_{1}, \ldots, W_{i}}^{2} \leq d_{i}, i=1, \ldots, L, \sigma_{X_{i}^{n} \mid W_{i}}^{2} \leq \delta_{i}$, $i=2, \ldots, L$, and $X_{i+1}^{n} \leftrightarrow X_{i}^{n} \leftrightarrow\left(W_{1}, \ldots, W_{i}\right)$ form a Markov chain, $i=1, \ldots, L-1$, where $d_{i} \in\left(0, \sigma_{X_{i}}^{2}\right]$, $i=1, \ldots, L$, and $\delta_{i} \in\left(0, \sigma_{X_{i}}^{2}\right], i=2, \ldots, L$.

\section{Theorem 6 :}

$$
\sup _{\mathcal{P}} \inf _{\mathcal{Q}} \Phi=\inf _{\mathcal{Q}} \sup _{\mathcal{P}} \Phi
$$

The following extremal inequality is needed for the proof of Theorem 6. Its proof can be found in Appendix E.

Theorem 7: Let $N_{i}^{n}$ be a zero-mean Gaussian random vector with i.i.d. entries of positive variance $\sigma_{N_{i}}^{2}, i=1,2,3$. Let $d$ be an arbitrary positive real number. Then, for any random vector $S^{n}$ and random object $W$, jointly independent of $\left(N_{1}^{n}, N_{2}^{n}, N_{3}^{n}\right)$, such that $\sigma_{S^{n} \mid W}^{2} \leq d$, we have

$$
\begin{aligned}
& h\left(S^{n}+N_{3}^{n} \mid W\right)-h\left(S^{n}+N_{1}^{n} \mid W\right)-h\left(S^{n}+N_{2}^{n} \mid W\right) \\
& \leq \max _{\gamma \in[0, d]} \frac{n}{2} \log \left(2 \pi e\left(\gamma+\sigma_{N_{3}}^{2}\right)\right)-\frac{n}{2} \log \left(2 \pi e\left(\gamma+\sigma_{N_{1}}^{2}\right)\right) \\
& \quad-\frac{n}{2} \log \left(2 \pi e\left(\gamma+\sigma_{N_{2}}^{2}\right)\right) .
\end{aligned}
$$

Now we proceed to prove Theorem 6. It suffices to show that

$$
\sup _{\mathcal{P}} \inf _{\mathcal{Q}} \Phi \geq \inf _{\mathcal{Q}} \sup _{\mathcal{P}} \Phi
$$

Let $Z_{i}^{n}$ be a zero-mean Gaussian random vector with i.i.d. entries of positive variance $\sigma_{Z_{i}}^{2}, i=1, \ldots, L-1$; moreover, we assume $Z_{i}^{n}$ is independent of $\left(X_{i+1}^{n}, W_{1}, \ldots, W_{i+1}\right), i=$ $1, \ldots, L-1$. Let $V_{i}=X_{i+1}^{n}+Z_{i}^{n}, i=1, \ldots, L-1$. Note that

$$
\begin{aligned}
I( & \left.X_{i}^{n} ; W_{i} \mid W_{1}, \ldots, W_{i-1}, X_{i}^{n}+Z_{i-1}^{n}\right) \\
= & I\left(X_{i}^{n}+Z_{i-1}^{n}, X_{i}^{n} ; W_{i} \mid W_{1}, \ldots, W_{i-1}\right) \\
& -I\left(X_{i}^{n}+Z_{i-1}^{n} ; W_{i} \mid W_{1}, \ldots, W_{i-1}\right) \\
= & I\left(X_{i}^{n} ; W_{i} \mid W_{1}, \ldots, W_{i-1}\right) \\
& -I\left(X_{i}^{n}+Z_{i-1}^{n} ; W_{i} \mid W_{1}, \ldots, W_{i-1}\right), \quad i=2, \ldots, L .
\end{aligned}
$$

Therefore, we have

$$
\begin{aligned}
\Phi= & \mu_{1} I\left(X_{1}^{n} ; W_{1}\right)+\sum_{i=2}^{L} \mu_{i}\left(I\left(X_{i}^{n}+Z_{i-1}^{n} ; W_{i}\right)\right. \\
& +I\left(X_{i}^{n} ; W_{i} \mid W_{1}, \ldots, W_{i-1}\right) \\
& \left.-I\left(X_{i}^{n}+Z_{i-1}^{n} ; W_{i} \mid W_{1}, \ldots, W_{i-1}\right)\right) \\
= & \frac{\mu_{1} n}{2} \log \left(2 \pi e \sigma_{X_{1}}^{2}\right)-\mu_{1} h\left(X_{1}^{n} \mid W_{1}\right) \\
+ & \sum_{i=2}^{L} \mu_{i}\left(\frac{n}{2} \log \left(2 \pi e\left(\sigma_{X_{i}}^{2}+\sigma_{Z_{i-1}}^{2}\right)\right)\right. \\
& -h\left(X_{i}^{n}+Z_{i-1}^{n} \mid W_{i}\right)+h\left(X_{i}^{n} \mid W_{1}, \ldots, W_{i-1}\right) \\
& -h\left(X_{i}^{n} \mid W_{1}, \ldots, W_{i}\right) \\
& -h\left(X_{i}^{n}+Z_{i-1}^{n} \mid W_{1}, \ldots, W_{i-1}\right) \\
& \left.+h\left(X_{i}^{n}+Z_{i-1}^{n} \mid W_{1}, \ldots, W_{i}\right)\right) \\
= & \frac{\mu_{1} n}{2} \log \left(2 \pi e \sigma_{X_{1}}^{2}\right)-\mu_{1} h\left(X_{1}^{n} \mid W_{1}\right) \\
- & \mu_{2}\left(h\left(X_{2}^{n}+Z_{1}^{n} \mid W_{1}\right)-h\left(X_{2}^{n} \mid W_{1}\right)\right) \\
& L-1 \\
+ & \sum_{i=2}\left(\mu _ { i } \left(h\left(X_{i}^{n}+Z_{i-1}^{n} \mid W_{1}, \ldots, W_{i}\right)\right.\right. \\
& \left.-h\left(X_{i}^{n} \mid W_{1}, \ldots, W_{i}\right)\right) \\
& -\mu_{i+1}\left(h\left(X_{i+1}^{n}+Z_{i}^{n} \mid W_{1}, \ldots, W_{i}\right)\right. \\
& \left.\left.-h\left(X_{i+1}^{n} \mid W_{1}, \ldots, W_{i}\right)\right)\right) \\
+ & \frac{\mu_{L}}{n}\left(h\left(X_{L}^{n}+Z_{L-1}^{n} \mid W_{1}, \ldots, W_{L}\right)\right. \\
& \left.-h\left(X_{L}^{n} \mid W_{1}, \ldots, W_{L}\right)\right) \\
+ & \sum_{i=2}^{L} \mu_{i}\left(\frac{n}{2} \log \left(2 \pi e\left(\sigma_{X_{i}}^{2}+\sigma_{Z_{i-1}}^{2}\right)\right)\right. \\
& \left.-h\left(X_{i}^{n}+Z_{i-1}^{n} \mid W_{i}\right)\right) . \\
& -
\end{aligned}
$$

It can be shown [cf., (12), (13), (15), and (16)] that

$$
\begin{aligned}
& -\mu_{1} h\left(X_{1}^{n} \mid W_{1}\right)-\mu_{2}\left(h\left(X_{2}^{n}+Z_{1}^{n} \mid W_{1}\right)-h\left(X_{2}^{n} \mid W_{1}\right)\right) \\
& \geq \min _{\gamma_{1} \in\left[0, d_{1}\right]} \frac{-\mu_{1} n}{2} \log \left(2 \pi e \gamma_{1}\right) \\
& \quad-\frac{\mu_{2} n}{2} \log \left(\frac{\gamma_{1}+\sigma_{\Delta_{1}}^{2}+\sigma_{Z_{1}}^{2}}{\gamma_{1}+\sigma_{\Delta_{1}}^{2}}\right) \\
& \quad \mu_{i}\left(h\left(X_{i}^{n}+Z_{i-1}^{n} \mid W_{1}, \ldots, W_{i}\right)-h\left(X_{i}^{n} \mid W_{1}, \ldots, W_{i}\right)\right) \\
& \quad-\mu_{i+1}\left(h\left(X_{i+1}^{n}+Z_{i}^{n} \mid W_{1}, \ldots, W_{i}\right)\right. \\
& \left.\quad-h\left(X_{i+1}^{n} \mid W_{1}, \ldots, W_{i}\right)\right) \\
& \geq \min _{\gamma_{i} \in\left[0, d_{i}\right]} \frac{\mu_{i} n}{2} \log \left(\frac{\gamma_{i}+\sigma_{Z_{i-1}}^{2}}{\gamma_{i}}\right) \\
& \quad-\frac{\mu_{i+1} n}{2} \log \left(\frac{\gamma_{i}+\sigma_{\Delta_{i}}^{2}+\sigma_{Z_{i-1}}^{2}}{\gamma_{i}+\sigma_{\Delta_{i}}^{2}}\right), \quad i=2, \ldots, L-1
\end{aligned}
$$




$$
\begin{aligned}
& h\left(X_{L}^{n}+Z_{L-1}^{n} \mid W_{1}, \ldots, W_{L}\right)-h\left(X_{L}^{n} \mid W_{1}, \ldots, W_{L}\right) \\
& \geq \min _{\gamma_{L} \in\left[0, d_{L}\right]} \frac{n}{2} \log \left(\frac{\gamma_{L}+\sigma_{Z_{L-1}}^{2}}{\gamma_{L}}\right) \\
& -h\left(X_{i}^{n}+Z_{i-1}^{n} \mid W_{i}\right) \\
& \geq-\frac{n}{2} \log \left(2 \pi e\left(\delta_{i}+\sigma_{Z_{i-1}}^{2}\right)\right), \quad i=2, \ldots, L .
\end{aligned}
$$

Substituting (49)-(52) into (48) and setting $\sigma_{Z_{i}}^{2}=\left(\theta_{i}^{-1}-\right.$ $\left.\sigma_{X_{i+1}}^{-2}\right)^{-1}, i=1, \ldots, L-1$, yields

$$
\Phi \geq \min _{\gamma_{i} \in\left[0, d_{i}\right], i=1, \ldots, L} n \psi(\bar{\mu}, \bar{\gamma}, \bar{\delta}, \bar{\theta})
$$

which further implies

$$
\inf _{\mathcal{Q}} \Phi \geq \min _{\gamma_{i} \in\left[0, d_{i}\right], i=1, \ldots, L} n \psi(\bar{\mu}, \bar{\gamma}, \bar{\delta}, \bar{\theta})
$$

Therefore, we have

$$
\begin{aligned}
& \sup _{\mathcal{P}} \inf _{\mathcal{Q}} \Phi \\
& \geq \sup _{\sigma_{Z_{i}}^{2}>0, i=1, \ldots, L-1} \min _{\gamma_{i} \in\left[0, d_{i}\right], i=1, \ldots, L} n \\
& \times\left.\psi(\bar{\mu}, \bar{\gamma}, \bar{\delta}, \bar{\theta})\right|_{\theta_{i}=\left(\sigma_{X_{i+1}}^{-2}+\sigma_{Z_{i}}^{-2}\right)^{-1}, i=1, \ldots, L-1} \\
& =\sup _{\theta_{i} \in\left(0, \sigma_{X_{i+1}}^{2}\right), i=1, \ldots, L-1} \min _{\gamma_{i} \in\left[0, d_{i}\right], i=1, \ldots, L} n \psi(\bar{\mu}, \bar{\gamma}, \bar{\delta}, \bar{\theta}) \text {. }
\end{aligned}
$$

In view of (53) and Theorem 3, for the purpose of establishing (47), it suffices to prove that

$$
\begin{aligned}
& \inf _{\mathcal{Q}} \sup _{\mathcal{P}} \Phi \\
& \leq \inf _{\gamma_{i} \in\left(0, d_{i}\right), i=1, \ldots, L} \max _{\theta_{i} \in\left[0, \sigma_{X_{i+1}}^{2}\right], i=1, \ldots, L-1} n \psi(\bar{\mu}, \bar{\gamma}, \bar{\delta}, \bar{\theta}) .
\end{aligned}
$$

To this end, we shall show that given any $\bar{\gamma}$ with $\gamma_{i} \in\left(0, d_{i}\right)$, $i=1, \ldots, L$, there exists $\left(W_{1}, \ldots, W_{L}\right)$ such that
A1) $\sigma_{X_{i}^{n} \mid W_{1}, \ldots, W_{i}}^{2} \leq \gamma_{i}, i=1, \ldots, L$,
A2) $\sigma_{X_{i}^{n} \mid W_{i}}^{2} \leq \delta_{i}, i=2, \ldots, L$,

A3) $X_{i+1}^{n^{2}} \leftrightarrow X_{i}^{n} \leftrightarrow\left(W_{1}, \ldots, W_{i}\right)$ form a Markov chain, $i=1, \ldots, L-1$.

\section{Moreover}

$$
\sup _{\mathcal{P}} \Phi \leq \max _{\theta_{i} \in\left[0, \sigma_{X_{i+1}}^{2}\right], i=1, \ldots, L-1} n \psi(\bar{\mu}, \bar{\gamma}, \bar{\delta}, \bar{\theta})
$$

Let $\overline{\gamma^{\prime}}$ and $\overline{\delta^{\prime}}$ be as specified in Lemma 2 . Note that $\gamma_{i}^{\prime} \in\left(0, \gamma_{i}\right]$, $i=1, \ldots, L, \delta_{i}^{\prime} \in\left(0, \delta_{i}\right], i=2, \ldots, L$, and

$$
\begin{aligned}
& \max _{\theta_{i} \in\left[0, \sigma_{X_{i+1}}^{2}\right], i=1, \ldots, L-1} \psi\left(\bar{\mu}, \overline{\gamma^{\prime}}, \overline{\delta^{\prime}}, \bar{\theta}\right) \\
& \leq \max _{\theta_{i} \in\left[0, \sigma_{X_{i+1}}^{2}\right], i=1, \ldots, L-1} \psi(\bar{\mu}, \bar{\gamma}, \bar{\delta}, \bar{\theta}) .
\end{aligned}
$$

As shown in Section IV, one can construct a zero-mean random vector $\left(U_{1}, \ldots, U_{L}, \tilde{U}_{1}, \ldots, \tilde{U}_{L}\right)$ jointly Gaussian with $\left(X_{1}, \ldots, X_{L}\right)$ such that:
B1) $\tilde{U}_{i}=\mathbb{E}\left[X_{i} \mid U_{1}, \ldots, U_{i}\right]$ and $\mathbb{E}\left[\left(X_{i}-\tilde{U}_{i}\right)^{2}\right]=\gamma_{i}^{\prime}$, $i=1, \ldots, L$,

B2) $\mathbb{E}\left[U_{i}^{2}\right]=\mathbb{E}\left[X_{i} U_{i}\right]=\sigma_{X_{i}}^{2}-\delta_{i}^{\prime}, i=2, \ldots, L$,

B3) $\left(X_{2}, \ldots, X_{L}\right) \leftrightarrow X_{1} \leftrightarrow U_{1}$ form a Markov chain, and $\left(X_{j}\right)_{j \neq i} \leftrightarrow\left(X_{i}, U_{1}, \ldots, U_{i-1}\right) \leftrightarrow U_{i}$ form a Markov chain, $i=1, \ldots, L$.

First assume that $\delta_{i}^{\prime}<\sigma_{X_{i}}^{2}, i=2, \ldots, L$. Define

$$
\begin{aligned}
& N_{i}=\frac{\sigma_{X_{i}}^{2}}{\sigma_{X_{i}}^{2}-\gamma_{i-1}^{\prime}-\sigma_{\Delta_{i-1}}^{2}} \tilde{U}_{i-1}-X_{i}, \quad i=2, \ldots, L \\
& \hat{N}_{i}=\frac{\sigma_{X_{i}}^{2}}{\sigma_{X_{i}}^{2}-\delta_{i}^{\prime}} U_{i}-X_{i}, \quad i=2, \ldots, L \\
& \tilde{N}_{i}=\frac{\sigma_{X_{i}}^{2}}{\sigma_{X_{i}}^{2}-\gamma_{i}^{\prime}} \tilde{U}_{i}-X_{i}, \quad i=2, \ldots, L .
\end{aligned}
$$

It can be verified that

$$
\begin{aligned}
\sigma_{N_{i}}^{2} & =\frac{\sigma_{X_{i}}^{2}\left(\gamma_{i-1}^{\prime}+\sigma_{\Delta_{i-1}}^{2}\right)}{\sigma_{X_{i}}^{2}-\gamma_{i-1}^{\prime}-\sigma_{\Delta_{i-1}}^{2}}, \quad i=2, \ldots, L \\
\sigma_{\hat{N}_{i}}^{2} & =\frac{\sigma_{X_{i}}^{2} \delta_{i}^{\prime}}{\sigma_{X_{i}}^{2}-\delta_{i}^{\prime}}, \quad i=2, \ldots, L \\
\sigma_{\tilde{N}_{i}}^{2} & =\frac{\sigma_{X_{i}}^{2} \gamma_{i}^{\prime}}{\sigma_{X_{i}}^{2}-\gamma_{i}^{\prime}}, \quad i=2, \ldots, L .
\end{aligned}
$$

Let $\left(X_{i j}, U_{i j}, \tilde{U}_{i j}, N_{i j}, \hat{N}_{i j}, \tilde{N}_{i j}, i=1, \ldots, L\right)_{j=1}^{n}$ be $n$ i.i.d. copies of $\left(X_{i}, U_{i}, \tilde{U}_{i}, N_{i}, \hat{N}_{i}, \tilde{N}_{i}, i=1, \ldots, L\right)$, and let $W_{i}=U_{i}^{n}, i=1, \ldots, L$. It is easy to see that A1), A2), and A3) are implied by B1), B2), and B3), respectively. Moreover, one can readily verify that $\left(N_{i, 1}^{n}, N_{i, 2}^{n}, N_{i, 3}^{n}\right)$ is independent of $\left(X_{i}^{n}, V_{i-1}\right), i=2, \ldots, L$. Note that

$$
\begin{aligned}
& \Phi= \mu_{1} I\left(X_{1}^{n} ; U_{1}^{n}\right)+\sum_{i=2}^{L} \mu_{i}\left(I\left(V_{i-1} ; U_{i}^{n}\right)\right. \\
&\left.+I\left(X_{i}^{n} ; U_{i}^{n} \mid U_{1}^{n}, \ldots, U_{i-1}^{n}, V_{i-1}\right)\right) \\
&= \mu_{1} I\left(X_{1}^{n} ; U_{1}^{n}\right)+\sum_{i=2}^{L} \mu_{i}\left(I\left(V_{i-1} ; U_{i}^{n}\right)\right. \\
&+I\left(X_{i}^{n} ; U_{i}^{n} \mid U_{1}^{n}, \ldots, U_{i-1}^{n}\right) \\
&\left.\quad-I\left(V_{i-1} ; U_{i}^{n} \mid U_{1}^{n}, \ldots, U_{i-1}^{n}\right)\right) \\
&=\mu_{1} I\left(X_{1}^{n} ; U_{1}^{n}\right)+\sum_{i=2}^{L} \mu_{i}\left(I\left(V_{i-1} ; U_{i}^{n}\right)\right. \\
& \quad+I\left(X_{i}^{n} ; U_{1}^{n}, \ldots, U_{i}^{n}\right)-I\left(X_{i}^{n} ; U_{1}^{n}, \ldots, U_{i-1}^{n}\right) \\
&\left.\quad-I\left(V_{i-1} ; U_{1}^{n}, \ldots, U_{i}^{n}\right)+I\left(V_{i-1} ; U_{1}^{n}, \ldots, U_{i-1}^{n}\right)\right) \\
&=\mu_{1} I\left(X_{1}^{n} ; U_{1}^{n}\right)+\sum_{i=2}^{L} \mu_{i}\left(I\left(V_{i-1} ; U_{i}^{n}\right)+I\left(X_{i}^{n} ; \tilde{U}_{i}^{n}\right)\right. \\
&\left.\quad-I\left(X_{i}^{n} ; \tilde{U}_{i-1}^{n}\right)-I\left(V_{i-1} ; \tilde{U}_{i}^{n}\right)+I\left(V_{i-1} ; \tilde{U}_{i-1}^{n}\right)\right) \\
&\left.\quad+I\left(V_{i-1} ; U_{i}^{n}\right)-I\left(V_{i-1} ; \tilde{U}_{i}^{n}\right)+I\left(V_{i-1} ; \tilde{U}_{i-1}^{n}\right)\right) \\
& \mu_{1} n \\
& 2
\end{aligned}
$$


We have

$$
\begin{aligned}
& I\left(V_{i-1} ; U_{i}^{n}\right)-I\left(V_{i-1} ; \tilde{U}_{i}^{n}\right)+I\left(V_{i-1} ; \tilde{U}_{i-1}^{n}\right) \\
& =I\left(V_{i-1} ; X_{i}^{n}+\hat{N}_{i}^{n}\right)-I\left(V_{i-1} ; X_{i}^{n}+\tilde{N}_{i}^{n}\right) \\
& \quad+I\left(V_{i-1} ; X_{i}^{n}+N_{i}^{n}\right) \\
& =\frac{n}{2} \log \left(2 \pi e\left(\sigma_{X_{i}}^{2}+\sigma_{N_{i}}^{2}\right)\right)+\frac{n}{2} \log \left(2 \pi e\left(\sigma_{X_{i}}^{2}+\sigma_{\hat{N}_{i}}^{2}\right)\right) \\
& \quad-\frac{n}{2} \log \left(2 \pi e\left(\sigma_{X_{i}}^{2}+\sigma_{\tilde{N}_{i}}^{2}\right)\right)+h\left(X_{i}^{n}+\tilde{N}_{i}^{n} \mid V_{i-1}\right) \\
& -h\left(X_{i}^{n}+N_{i}^{n} \mid V_{i-1}\right)-h\left(X_{i}^{n}+\hat{N}_{i}^{n} \mid V_{i-1}\right) \\
& \quad i=2, \ldots, L
\end{aligned}
$$

Since $\sigma_{X_{i}^{n} \mid V_{i-1}}^{2} \leq \sigma_{X_{i}}^{2}$, it follows from Theorem 7 that

$$
\begin{aligned}
& h\left(X_{i}^{n}+\tilde{N}_{i}^{n} \mid V_{i-1}\right)-h\left(X_{i}^{n}+N_{i}^{n} \mid V_{i-1}\right) \\
& \quad-h\left(X_{i}^{n}+\hat{N}_{i}^{n} \mid V_{i-1}\right) \\
& \leq \max _{\theta_{i-1} \in\left[0, \sigma_{X_{i}}^{2}\right]} \frac{n}{2} \log \left(2 \pi e\left(\theta_{i-1}+\sigma_{\tilde{N}_{i}}^{2}\right)\right) \\
& \quad-\frac{n}{2} \log \left(2 \pi e\left(\theta_{i-1}+\sigma_{N_{i}}^{2}\right)\right)-\frac{n}{2} \log \left(2 \pi e\left(\theta_{i-1}+\sigma_{\hat{N}_{i}}^{2}\right)\right), \\
& i=2, \ldots, L .
\end{aligned}
$$

Therefore, we have

$$
\begin{gathered}
I\left(V_{i-1} ; U_{i}^{n}\right)-I\left(V_{i-1} ; \tilde{U}_{i}^{n}\right)+I\left(V_{i-1} ; \tilde{U}_{i-1}^{n}\right) \\
\leq \frac{n}{2} \log \left(\frac{\left(\sigma_{X_{i}}^{2}+\sigma_{N_{i}}^{2}\right)\left(\sigma_{X_{i}}^{2}+\sigma_{\hat{N}_{i}}^{2}\right)\left(\theta_{i-1}+\sigma_{\tilde{N}_{i}}^{2}\right)}{\left(\theta_{i-1}+\sigma_{N_{i}}^{2}\right)\left(\theta_{i-1}+\sigma_{\hat{N}_{i}}^{2}\right)\left(\sigma_{X_{i}}^{2}+\sigma_{\tilde{N}_{i}}^{2}\right)}\right) \\
i=2, \ldots, L .
\end{gathered}
$$

Substituting (60) into (59) and invoking (56)-(58) yields

$$
\Phi \leq \max _{\theta_{i} \in\left[0, \sigma_{X_{i+1}}^{2} \max _{i=1, \ldots, L-1}\right.} n \psi\left(\bar{\mu}, \overline{\gamma^{\prime}}, \overline{\delta^{\prime}}, \bar{\theta}\right)
$$

If $\delta_{i}^{\prime}=\sigma_{X_{i}}^{2}$ for some $i$, then

$$
I\left(V_{i-1} ; U_{i}^{n}\right)-I\left(V_{i-1} ; \tilde{U}_{i}^{n}\right)+I\left(V_{i-1} ; \tilde{U}_{i-1}^{n}\right)=0 .
$$

As a consequence, one can readily verify that (61) continues to hold. Combining (55) and (61) gives

$$
\Phi \leq \max _{\theta_{i} \in\left[0, \sigma_{X_{i+1}}^{2}\right], i=1, \ldots, L-1} n \psi(\bar{\mu}, \bar{\gamma}, \bar{\delta}, \bar{\theta}) .
$$

which further implies (54). This completes the proof of Theorem 6.

\section{Minimum Sum Rate: A Special Case}

In this section, we focus on the case $\bar{\mu}=(1, \ldots, 1)$ (which corresponds to the sum rate). Let $\sigma_{X_{i}}^{2}=\rho^{i-1}, i=1, \ldots, L$, and $\sigma_{\Delta_{i}}^{2}=\rho^{i-1}(\rho-1), i=1, \ldots, L-1$, where $\rho>1$. Moreover, let $\bar{d}=\left(\rho^{0} d, \ldots, \rho^{L-1} d\right)$ and $\bar{\delta}=\left(\rho \delta, \ldots, \rho^{L-1} \delta\right)$, where $d \in(0,1]$ and $\delta \in(0,1]$. By Theorem $3, \kappa_{l}(\bar{\mu}, \bar{d}, \bar{\delta})$ and $\kappa_{u}(\bar{\mu}, \bar{d}, \bar{\delta})$ coincide in this special case; therefore, we shall denote them by $\kappa(\bar{\mu}, \bar{d}, \bar{\delta})$. The main result of this section is an explicit characterization of $\kappa(\bar{\mu}, \bar{d}, \bar{\delta})$.
Theorem 8:

1) If $d \geq\left(\frac{\rho}{d+\rho-1}+\frac{1}{\delta}-1\right)^{-1}$, then

$$
\kappa(\bar{\mu}, \bar{d}, \bar{\delta})=\frac{1}{2} \log \left(\frac{1}{d}\right)+\frac{L-1}{2} \log \left(\frac{1}{\delta}\right) .
$$

2) If $d \leq \frac{\rho \delta-1}{\rho-1}$, then

$$
\kappa(\bar{\mu}, \bar{d}, \bar{\delta})=\frac{1}{2} \log \left(\frac{1}{d}\right)+\frac{L-1}{2} \log \left(\frac{d+\rho-1}{\rho d}\right) .
$$

3) If $\frac{\rho \delta-1}{\rho-1}<d<\left(\frac{\rho}{d+\rho-1}+\frac{1}{\delta}-1\right)^{-1}$, then

$$
\begin{aligned}
& \kappa(\bar{\mu}, \bar{d}, \bar{\delta}) \\
& =\frac{1}{2} \log \left(\frac{1}{d}\right)+\frac{L-1}{2} \log \left(\frac{(d+\rho-1)}{((1-\theta)(d+\rho-1)+\rho \theta)}\right. \\
& \left.\quad \times \frac{((1-\theta) d+\theta)}{((1-\theta) \delta+\theta) d}\right)
\end{aligned}
$$

where

$$
\theta=\sqrt{\frac{\rho-1}{1-d}\left(\frac{\delta}{1-\delta}-\frac{d}{1-d}\right)}-\frac{d}{1-d} .
$$

Proof:

1) Let $\overline{\gamma^{*}}=\bar{d}$ and $\overline{\theta^{*}}=(0, \ldots, 0)$. It can be verified that $\left(\overline{\gamma^{*}}, \overline{\theta^{*}}\right)$ satisfies (38) and (39) (see the optimality conditions in Lemmas 1, 4, 5, and 6). Therefore, it follows by the proof of Theorem 3 that

$$
\begin{aligned}
\kappa(\bar{\mu}, \bar{d}, \bar{\delta}) & =\psi\left(\bar{\mu}, \overline{\gamma^{*}}, \bar{\delta}, \overline{\theta^{*}}\right) \\
& =\frac{1}{2} \log \left(\frac{1}{d}\right)+\frac{L-1}{2} \log \left(\frac{1}{\delta}\right) .
\end{aligned}
$$

Note that in this case, we can decrease the hierarchical distortion constraint $\bar{d}$ to $\overline{d^{\prime}} \triangleq\left(d_{1}^{\prime}, \ldots, d_{L}^{\prime}\right)$ without affecting the minimum sum rate, where

$$
\begin{aligned}
& d_{1}^{\prime}=d \\
& d_{i}^{\prime}=\rho^{i-1}\left(\frac{\rho^{i-1}}{d_{i-1}^{\prime}+\rho^{i-2}(\rho-1)}+\frac{1}{\delta}-1\right)^{-1}, \\
& \quad i=2, \ldots, L .
\end{aligned}
$$

2) Let $\overline{\gamma^{*}}=\bar{d}$ and $\overline{\theta^{*}}=\left(\rho, \ldots, \rho^{L-1}\right)$. It can be verified that $\left(\overline{\gamma^{*}}, \overline{\theta^{*}}\right)$ satisfies (38) and (39) (see the optimality conditions in Lemmas 1, 4, 5, and 6). Therefore, it follows by the proof of Theorem 3 that

$$
\begin{aligned}
\kappa(\bar{\mu}, \bar{d}, \bar{\delta}) & =\psi\left(\bar{\mu}, \overline{\gamma^{*}}, \bar{\delta}, \overline{\theta^{*}}\right) \\
& =\frac{1}{2} \log \left(\frac{1}{d}\right)+\frac{L-1}{2} \log \left(\frac{d+\rho-1}{\rho d}\right) .
\end{aligned}
$$

Note that in this case, we can decrease the individual distortion constraint $\bar{\delta}$ to $\overline{\delta^{\prime}} \triangleq\left(\delta_{2}^{\prime}, \ldots, \delta_{L}^{\prime}\right)$ without affecting the minimum sum rate, where

$$
\delta_{i}^{\prime}=\rho^{i-2}(\rho-1) d+\rho^{i-2}, \quad i=2, \ldots, L .
$$

3) Let $\overline{\gamma^{*}}=\bar{d}$ and $\overline{\theta^{*}}=\left(\rho \theta, \ldots, \rho^{L-1} \theta\right)$. It is easy to verify that $\left(\overline{\gamma^{*}}, \overline{\theta^{*}}\right)$ satisfies (39) (see the optimality condition in 
Lemma 1). Note that the optimality conditions in Lemmas 4 and 6 are clearly satisfied; therefore, to verify (38), it suffices to show that $\left(\overline{\gamma^{*}}, \overline{\theta^{*}}\right)$ satisfies the optimality condition in Lemma 5. In view of the fact that $\theta \in(0,1)$ and that

$$
\tilde{a}_{i}=\rho^{3 i-2}(\rho-1)(1-\theta) \theta>0
$$

we just need to show that $\hat{\gamma}_{i} \geq \rho^{i-1} d$, where

$$
\hat{\gamma}_{i}=\rho^{i-1}\left(1+\sqrt{\frac{\rho}{1-\theta}}\right), \quad i=2, \ldots, L-1 .
$$

This is indeed true since $1+\sqrt{\frac{\rho}{1-\theta}}>1 \geq d$. Therefore, it follows by the proof of Theorem 3 that

$$
\begin{aligned}
& \kappa(\bar{\mu}, \bar{d}, \bar{\delta}) \\
& =\psi\left(\bar{\mu}, \overline{\gamma^{*}}, \bar{\delta}, \overline{\theta^{*}}\right) \\
& =\frac{1}{2} \log \left(\frac{1}{d}\right)+\frac{L-1}{2} \log \left(\frac{(d+\rho-1)}{((1-\theta)(d+\rho-1)+\rho \theta)}\right. \\
& \left.\quad \times \frac{((1-\theta) d+\theta)}{((1-\theta) \delta+\theta) d}\right) .
\end{aligned}
$$

\section{Miscellaneous Results}

\section{A. Robust Predictive Coding System}

In this section, we propose an efficient implementation of the robust predictive coding scheme associated with Lemma 3. For simplicity, throughout this section, we describe the scheme in the form of single-letter operations; however, it should be understood that in fact such a scheme has to be implemented over long blocks in order to approach the information-theoretic limits.

As shown in Section IV, to minimize the weighted sum rate $\bar{\mu} \bar{R}^{T}$ of the robust predictive coding scheme associated with Lemma 3 , there is no loss of optimality in considering zero-mean random vector $\left(U_{1}, \ldots, U_{L}, \tilde{U}_{1}, \ldots, \tilde{U}_{L}\right)$ jointly Gaussian with $\left(X_{1}, \ldots, X_{L}\right)$ such that

$$
\begin{aligned}
& \mathbb{E}\left[U_{1}^{2}\right]=\mathbb{E}\left[X_{1} U_{1}\right]=\sigma_{X_{1}}^{2}-\gamma_{1}^{\prime} \\
& \mathbb{E}\left[U_{i}^{2}\right]=\mathbb{E}\left[X_{i} U_{i}\right]=\sigma_{X_{i}}^{2}-\delta_{i}^{\prime}, \quad i=2, \ldots, L \\
& \mathbb{E}\left[\tilde{U}_{i-1} U_{i}\right]=\xi_{i-1}, \quad i=2, \ldots, L \\
& \tilde{U}_{1}=\mathbb{E}\left[X_{1} \mid U_{1}\right]=U_{1} \\
& \tilde{U}_{i}=\mathbb{E}\left[X_{i} \mid U_{1}, \ldots, U_{i}\right]=\mathbb{E}\left[X_{i} \mid \tilde{U}_{i-1}, U_{i}\right] \\
& \quad=a_{i-1} \tilde{U}_{i-1}+b_{i-1} U_{i}, \quad i=2, \ldots, L \\
& \mathbb{E}\left[\left(X_{i}-\tilde{U}_{i}\right)^{2}\right]=\gamma_{i}^{\prime}, \quad i=1, \ldots, L \\
& \mathbb{E}\left[\left(X_{i}-U_{i}\right)^{2}\right]=\delta_{i}^{\prime}, \quad i=2, \ldots, L
\end{aligned}
$$

where $\overline{\gamma^{\prime}}$ and $\overline{\delta^{\prime}}$ satisfy

$$
\begin{aligned}
& 0<\gamma_{i}^{\prime} \leq d_{i}, \quad i=1, \ldots, L \\
& 0<\delta_{i}^{\prime} \leq \delta_{i}, \quad i=2, \ldots, L \\
& \gamma_{i}^{\prime}+\sigma_{\Delta_{i}}^{2}+\delta_{i+1}^{\prime}-\sigma_{X_{i+1}}^{2} \\
& \leq \gamma_{i+1}^{\prime} \leq\left(\frac{1}{\gamma_{i}^{\prime}+\sigma_{\Delta_{i}}^{2}}+\frac{1}{\delta_{i+1}^{\prime}}-\frac{1}{\sigma_{X_{i+1}}^{2}}\right)^{-1} \\
& \quad i=1, \ldots, L-1
\end{aligned}
$$

and the parameters $\xi_{i}, a_{i}$, and $b_{i}, i=1, \ldots, L-1$ are defined in (23)-(25), respectively; moreover, $\left(X_{2}, \ldots, X_{L}\right) \leftrightarrow X_{1} \leftrightarrow U_{1}$ form a Markov chain, and $\left(\left(X_{j}\right)_{j \neq i}, U_{1}, \ldots, U_{i-1}\right) \leftrightarrow$ $\left(X_{i}, \tilde{U}_{i-1}\right) \leftrightarrow U_{i}$ form a Markov chain, $i=2, \ldots, L$. Let

$$
\begin{aligned}
& R_{1}=I\left(X_{1} ; U_{1}\right) \\
& R_{i}=I\left(X_{i}, U_{1}, \ldots, U_{i-1} ; U_{i}\right), \quad i=2, \ldots, L
\end{aligned}
$$

where $R_{i}$ is the rate of Encoder $i$. One can interpret $X_{1}$ and $U_{1}$, respectively, as the input and the output of Encoder 1; similarly, $\left(X_{i}, U_{1}, \ldots, U_{i-1}\right)$ and $U_{i}$ can be interpreted, respectively, as the input and the output of Encoder $i, i=2, \ldots, L$. Given the outputs from the first $i$ encoders, the decoder can compute $\tilde{U}_{i}$ and use it as the reconstruction of $X_{i}$, and the resulting distortion is $\gamma_{i}^{\prime}, i=1, \ldots, L$. If the decoder only receives the output from Encoder $i$, then it simply uses $U_{i}$ as the reconstruction of $X_{i}$, and the resulting distortion is $\delta_{i}^{\prime}, i=2, \ldots, L$. Moreover, in view of the fact that

$$
I\left(X_{i}, U_{1}, \ldots, U_{i-1} ; U_{i}\right)=I\left(X_{i}, \tilde{U}_{i-1} ; U_{i}\right)
$$

it suffices to provide Encoder $i$ with $\left(X_{i}, \tilde{U}_{i-1}\right)$ as the input, $i=2, \ldots, L$. Note that we can write

$$
\begin{gathered}
U_{1}=\mathbb{E}\left[U_{1} \mid X_{1}\right]+N_{1}=\alpha_{1} X_{1}+N_{1} \\
U_{i}=\mathbb{E}\left[U_{i} \mid X_{i}, \tilde{U}_{i-1}\right]+N_{i}=\alpha_{i} X_{i}+\beta_{i-1} \tilde{U}_{i-1}+N_{i}, \\
i=2, \ldots, L
\end{gathered}
$$

where

$$
\begin{aligned}
& \alpha_{1}=\frac{\sigma_{X_{1}}^{2}-\gamma_{1}^{\prime}}{\sigma_{X_{1}}^{2}} \\
& \alpha_{i}=\frac{\sigma_{X_{i}}^{2}-\delta_{i}^{\prime}-\xi_{i-1}}{\gamma_{i-1}^{\prime}+\sigma_{\Delta_{i-1}}^{2},} \quad i=2, \ldots, L \\
& \beta_{i}= \begin{cases}0, & \gamma_{i}^{\prime}=\sigma_{X_{i}}^{2} \\
\frac{\xi_{i}}{\sigma_{X_{i}}^{2}-\gamma_{i}^{\prime}}-\frac{\sigma_{X_{i+1}}^{2}-\delta_{i+1}^{\prime}-\xi_{i}}{\gamma_{i}^{\prime}+\sigma_{\Delta_{i}}^{2}}, & \text { otherwise }\end{cases} \\
& \mathbb{E}\left[N_{1}^{2}\right]=\frac{\left(\sigma_{X_{1}}^{2}-\gamma_{1}^{\prime}\right) \gamma_{1}^{\prime}}{\sigma_{X_{1}}^{2}} \\
& \mathbb{E}\left[N_{i}^{2}\right]=\left\{\begin{array}{rr}
\frac{\left(\sigma_{X_{i}}^{2}-\delta_{i}^{\prime}\right) \delta_{i}^{\prime}}{\sigma_{X_{i}}^{2}}, & \gamma_{i-1}^{\prime}=\sigma_{X_{i-1}}^{2} \\
\sigma_{X_{i}}^{2}-\delta_{i}^{\prime}-\frac{\left(\sigma_{X_{i}}^{2}-\delta_{i}^{\prime}-\xi_{i-1}\right)^{2}}{\gamma_{i-1}^{\prime}+\sigma_{\Delta_{i-1}}^{2}}-\frac{\xi_{i-1}^{2}}{\sigma_{X_{i-1}-\gamma_{i-1}^{\prime}}^{2}} & \text { otherwise }
\end{array}\right.
\end{aligned}
$$

It is clear that

$$
\begin{aligned}
& I\left(X_{1} ; U_{1}\right)=I\left(\alpha_{1} X_{1} ; \alpha_{1} X_{1}+N_{1}\right) \\
& I\left(X_{i}, \tilde{U}_{i-1} ; U_{i}\right) \\
& =I\left(\alpha_{i} X_{i}+\beta_{i-1} \tilde{U}_{i-1} ; \alpha_{i} X_{i}+\beta_{i-1} \tilde{U}_{i-1}+N_{i}\right), \\
& i=2, \ldots, L .
\end{aligned}
$$

As a consequence, we can interpret Encoder 1 as a quantizer with $\alpha_{1} X_{1}, U_{1}$, and $N_{1}$ respectively as the input, the output, and the quantization error; similarly, we can interpret Encoder $i$ as a quantizer with $\alpha_{i} X_{i}+\beta_{i-1} \tilde{U}_{i-1}, U_{i}$, and $N_{i}$ respectively 


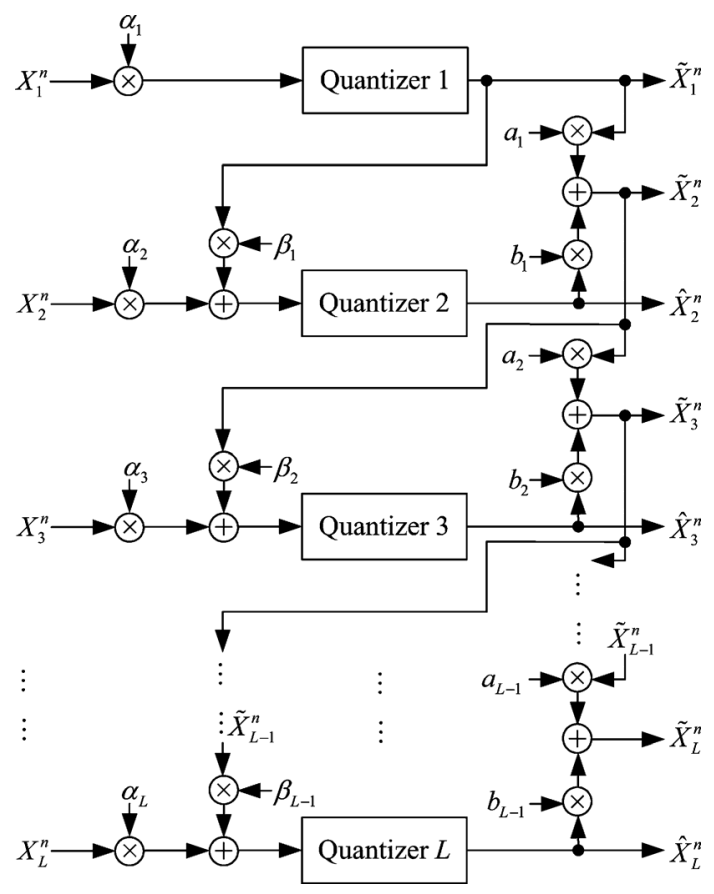

Fig. 3. Robust predictive coding via successive quantization. Here $\tilde{X}_{i}^{n}$ can be viewed as a multiletter version of $\tilde{U}_{i}, i=1, \ldots, L$, and $\hat{X}_{i}^{n}$ can be viewed as a multiletter version of $U_{i}, i=2, \ldots, L$.

as the input, the output, and the quantization error, $i=2, \ldots, L$. Furthermore, in view of the fact that

$$
\tilde{U}_{i}=a_{i-1} \tilde{U}_{i-1}+b_{i-1} U_{i}, \quad i=2, \ldots, L
$$

the calculation of $\left(\tilde{U}_{1}, \ldots, \tilde{U}_{L}\right)$ at the encoders and the decoder can be performed iteratively. A robust predictive coding system based on this interpretation is depicted in Fig. 3. It is worth mentioning that one can implement the quantization operation in such a system by using entropy-coded dithered lattice quantizers (see, e.g., [10]-[12]).

\section{B. Reconstruction Based on an Arbitrary Subset of Encoder Outputs}

As pointed out in Section VIII-A, one can interpret $U_{i}$ as the output of Encoder $i, i=1, \ldots, L$, for the robust predictive coding scheme associated with Lemma 3. Although it is developed for the scenario where only the hierarchical distortion constraint and the individual distortion constraint are imposed, this scheme has a desirable property that every subset of the encoder outputs is decodable. For example, if at the time of reconstructing $X_{5}$, the decoder only receives the outputs from a subset of the first 5 encoders (say, $\left(U_{1}, U_{3}, U_{4}\right)$ ), then it can still decode these outputs and further use $\mathbb{E}\left[X_{5} \mid U_{1}, U_{3}, U_{4}\right]$ as the reconstruction of $X_{5}$ (with the resulting distortion equal to $\left.\sigma_{X_{5} \mid U_{1}, U_{3}, U_{4}}^{2}\right)$.

Now we proceed to give a detailed analysis for this kind of scenario. Again we shall focus on the case where $\left(U_{1}, \ldots, U_{L}\right)$ satisfies the conditions listed in Section VIII-A. Assume that the decoder receives $U_{j}, j \in \mathcal{A}$ for some non-empty set $\mathcal{A} \subseteq$
$\{1, \ldots, L\}$; moreover, at the time of reconstructing $X_{i}$, the decoder is only allowed to use $\left(U_{j}\right)_{j \in \mathcal{A}, j \leq i}$. With no loss of generality, we shall assume $\mathbb{E}\left[\left(U_{i}^{2}\right)\right] \neq 0$ (which implies $\delta_{i}^{\prime}<\sigma_{X_{i}}^{2}$ ) for all $i \in \mathcal{A}$.

Define

$$
\begin{aligned}
& \hat{U}_{i}=\mathbb{E}\left[X_{i} \mid\left(U_{j}\right)_{j \in \mathcal{A}, j \leq i}\right], \quad i=1, \ldots, L \\
& \hat{d}_{i}=\mathbb{E}\left[\left(X_{i}-\hat{U}_{i}\right)^{2}\right], \quad i=1, \ldots, L .
\end{aligned}
$$

Note that $\hat{U}_{i}=0$ and $\hat{d}_{i}=\sigma_{X_{i}}^{2}$ if $j>i$ for all $j \in \mathcal{A}$. We shall show that $\left(\hat{U}_{1}, \ldots, \hat{U}_{L}\right)$ and $\left(\hat{d}_{1}, \ldots, \hat{d}_{L}\right)$ can be computed iteratively.

It is clear that

$$
\begin{aligned}
& \hat{U}_{1}= \begin{cases}0, & 1 \notin \mathcal{A} \\
U_{1}, & \text { otherwise }\end{cases} \\
& \hat{d}_{1}= \begin{cases}\sigma_{X_{1}}^{2}, & 1 \notin \mathcal{A} \\
\gamma_{1}^{\prime}, & \text { otherwise. }\end{cases}
\end{aligned}
$$

For $i=2, \ldots, L$, we have $\hat{U}_{i}=\hat{U}_{i-1}$ and $\hat{d}_{i}=\hat{d}_{i-1}+$ $\sigma_{\Delta_{i-1}}^{2}$ if $i \notin \mathcal{A}$; moreover, we have $\hat{U}_{i}=U_{i}$ and $\hat{d}_{i}=\delta_{i}^{\prime}$ if $\hat{d}_{i-1}=\sigma_{X_{i-1}}^{2}$. Therefore, it suffices to consider the case where $i \in \mathcal{A}$ and $\hat{d}_{i-1}<\sigma_{X_{i-1}}^{2}$ (which implies $\gamma_{i-1}^{\prime}<\sigma_{X_{i-1}}^{2}$ ). Since $\left(U_{1}, \ldots, U_{i-1}\right) \leftrightarrow \tilde{U}_{i-1} \leftrightarrow\left(X_{i}, \tilde{U}_{i-1}\right) \leftrightarrow U_{i}$ form a Markov chain, it follows that $\left(U_{j}\right)_{j \in \mathcal{A}, j<i} \leftrightarrow \hat{U}_{i-1} \leftrightarrow \tilde{U}_{i-1} \leftrightarrow$ $\left(X, \tilde{U}_{i}\right) \leftrightarrow U_{i}$ form a Markov chain. As a consequence

$$
\hat{U}_{i}=\mathbb{E}\left[X_{i} \mid \hat{U}_{i-1}, U_{i}\right]
$$

In view of the fact that

$$
\mathbb{E}\left[\hat{U}_{i-1}^{2}\right]=\mathbb{E}\left[\tilde{U}_{i-1} \hat{U}_{i-1}\right]=\sigma_{X_{i-1}}^{2}-\hat{d}_{i-1}
$$

we can write

$\hat{U}_{i-1}=\mathbb{E}\left[\hat{U}_{i-1} \mid \tilde{U}_{i-1}\right]+\hat{N}_{i-1}=\frac{\sigma_{X_{i-1}}^{2}-\hat{d}_{i-1}}{\sigma_{X_{i-1}}^{2}-\gamma_{i-1}^{\prime}} \tilde{U}_{i-1}+\hat{N}_{i-1}$

where $\hat{N}_{i-1}$ is independent of $\left(X_{i}, \tilde{U}_{i-1}, U_{i}\right)$ (recall that $\hat{U}_{i-1} \leftrightarrow \tilde{U}_{i-1} \leftrightarrow\left(X_{i}, U_{i}\right)$ form a Markov chain). Therefore

$$
\begin{aligned}
\mathbb{E}\left[\hat{U}_{i-1} U_{i}\right] & =\frac{\sigma_{X_{i-1}}^{2}-\hat{d}_{i-1}}{\sigma_{X_{i-1}}^{2}-\gamma_{i-1}^{\prime}} \mathbb{E}\left[\tilde{U}_{i-1} U_{i}\right] \\
& =\frac{\left(\sigma_{X_{i-1}}^{2}-\hat{d}_{i-1}\right) \xi_{i-1}}{\sigma_{X_{i-1}}^{2}-\gamma_{i-1}^{\prime}} .
\end{aligned}
$$

It is also easy to see that

$$
\mathbb{E}\left[X_{i} \hat{U}_{i-1}\right]=\sigma_{X_{i-1}}^{2}-\hat{d}_{i-1} .
$$

Now one can readily verify that

$$
\begin{aligned}
& \hat{U}_{i}=\hat{\alpha}_{i-1} \hat{U}_{i-1}+\hat{\beta}_{i-1} U_{i} \\
& \hat{d}_{i}=\sigma_{X_{i}}^{2}-\hat{\alpha}_{i-1}\left(\sigma_{X_{i-1}}^{2}-\hat{d}_{i-1}\right)-\hat{\beta}_{i-1}\left(\sigma_{X_{i}}^{2}-\delta_{i}^{\prime}\right)
\end{aligned}
$$




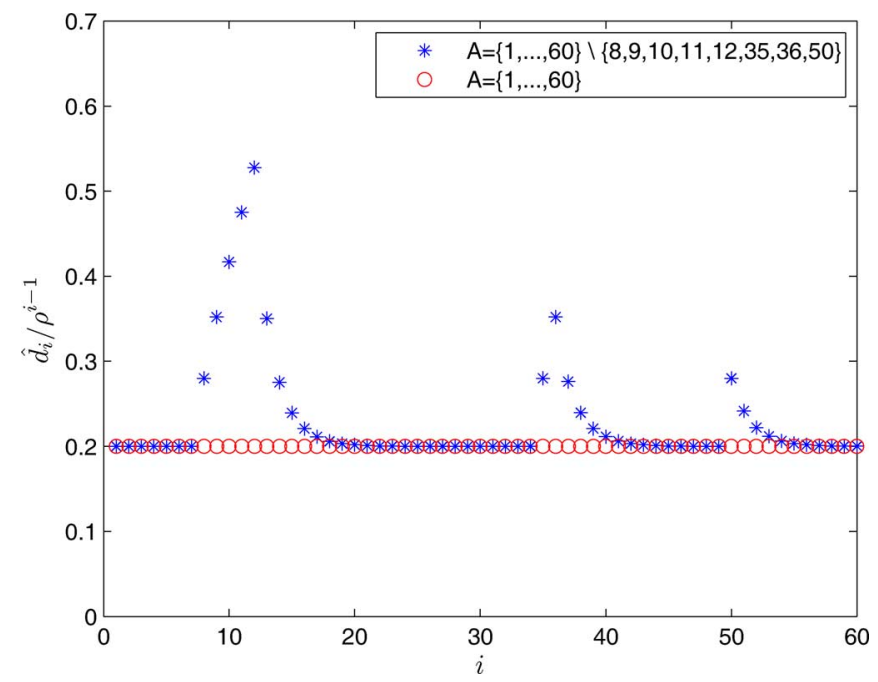

Fig. 4. Reconstruction based on a subset of encoder outputs.

where

$$
\begin{aligned}
\hat{\alpha}_{i-1}= & \frac{\left(\sigma_{X_{i-1}}^{2}-\gamma_{i-1}^{\prime}\right)^{2}\left(\sigma_{X_{i-1}}^{2}-\hat{d}_{i-1}\right)\left(\sigma_{X_{i}}^{2}-\delta_{i}^{\prime}\right)}{\chi_{i-1}} \\
& -\frac{\left(\sigma_{X_{i-1}}^{2}-\gamma_{i-1}^{\prime}\right)\left(\sigma_{X_{i-1}}^{2}-\hat{d}_{i-1}\right) \xi_{i-1}\left(\sigma_{X_{i}}^{2}-\delta_{i}^{\prime}\right)}{\chi_{i-1}} \\
\hat{\beta}_{i-1}= & -\frac{\left(\sigma_{X_{i-1}}^{2}-\gamma_{i-1}^{\prime}\right)\left(\sigma_{X_{i-1}}^{2}-\hat{d}_{i-1}\right)^{2} \xi_{i-1}}{\chi_{i-1}} \\
& +\frac{\left(\sigma_{X_{i-1}}^{2}-\gamma_{i-1}^{\prime}\right)^{2}\left(\sigma_{X_{i-1}}^{2}-\hat{d}_{i-1}\right)\left(\sigma_{X_{i}}^{2}-\delta_{i}^{\prime}\right)}{\chi_{i-1}}
\end{aligned}
$$

with

$$
\begin{aligned}
\chi_{i-1}= & \left(\sigma_{X_{i-1}}^{2}-\gamma_{i-1}^{\prime}\right)^{2}\left(\sigma_{X_{i-1}}^{2}-\hat{d}_{i-1}\right)\left(\sigma_{X_{i}}^{2}-\delta_{i}^{\prime}\right) \\
& -\left(\sigma_{X_{i-1}}^{2}-\hat{d}_{i-1}\right)^{2} \xi_{i-1}^{2} .
\end{aligned}
$$

An illustrative example is given in Fig. 4. In this example, we choose $\sigma_{X_{i}}^{2}=\rho^{i-1}, i=1, \ldots, L$, and $\sigma_{\Delta_{i}}^{2}=\rho^{i-1}(\rho-1)$, $i=1, \ldots, L-1$, where $L=60$ and $\rho=\frac{10}{9}$; moreover, we set $\overline{\gamma^{\prime}}=\left(\rho^{0} \gamma^{\prime}, \ldots, \rho^{L-1} \gamma^{\prime}\right)$ and $\overline{\delta^{\prime}}=\left(\rho \delta^{\prime}, \ldots, \rho^{L-1} \delta^{\prime}\right)$, where $\gamma^{\prime}=0.2$ and $\delta^{\prime}=0.5$. We plot $\hat{d}_{i} / \rho^{i-1}, i=1, \ldots, L$, for the scenario where $\mathcal{A}=\{1, \ldots, 60\} \backslash\{8,9,10,11,12,35,36,50\}$. A comparison with the ideal scenario (i.e., $\mathcal{A}=\{1, \ldots, 60\}$ ) shows that the proposed scheme has a desirable "self-recovery" property.

\section{CONCLUSION}

We have partially characterized the rate region of robust sequential coding and robust predictive coding for the Gauss-Markov source model under the mean squared error distortion constraint. More fundamentally, our investigation reveals an information-theoretic minimax theorem, which can be obtained by coupling two extremal inequalities. It is worth noting that most of the results in this study can be extended to the vector source setting in a relatively straightforward manner.
In particular, one can establish the vector version of Theorems 5 and 7 by leveraging techniques developed in [13]-[15].

\section{APPENDIX A \\ PROOF OF THEOREM 5}

The following lemma is well known.

Lemma 7: For any random vector $S^{n}$ and random object $W$ such that $\sigma_{S^{n} \mid W}^{2} \leq d$,

$$
h\left(S^{n} \mid W\right) \leq \frac{n}{2} \log (2 \pi e d) .
$$

The following result is a variant of the worst additive noise lemma by Ihara [16] as well as Diggavi and Cover [17, Lemma II.2]. Its proof can be found in [9, Appendix B].

Lemma 8: Let $Z^{n}$ be a zero-mean Gaussian random vector with i.i.d. entries of positive variance $\sigma_{Z}^{2}$. For any random vector $S^{n}$ and random object $W$, jointly independent of $Z^{n}$, such that $\sigma_{S^{n} \mid W}^{2} \leq d$,

$$
h\left(S^{n}+Z^{n} \mid W\right)-h\left(S^{n} \mid W\right) \geq \frac{n}{2} \log \left(\frac{d+\sigma_{Z}^{2}}{d}\right) .
$$

Lemma 9: Let $Z_{i}^{n}$ be a zero-mean Gaussian random vector with i.i.d. entries of positive variance $\sigma_{Z_{i}}^{2}, i=1,2$. Let $\nu_{1}$ and $\nu_{2}$ be arbitrary real numbers satisfying $\nu_{1} \geq \nu_{2} \geq 0$. Then for any random vector $S^{n}$ and random object $W$, jointly independent of $\left(Z_{1}^{n}, Z_{2}^{n}\right)$, such that $\sigma_{S^{n} \mid W}^{2} \leq d$,

$$
\begin{aligned}
& -\nu_{1} h\left(S^{n} \mid W\right)-\nu_{2}\left(h\left(S^{n}+Z_{2}^{n} \mid W\right)-h\left(S^{n}+Z_{1}^{n} \mid W\right)\right) \\
& \geq-\frac{\nu_{1} n}{2} \log (2 \pi e d)-\frac{\nu_{2} n}{2} \log \left(\frac{d+\sigma_{Z_{2}}^{2}}{d+\sigma_{Z_{1}}^{2}}\right) .
\end{aligned}
$$

Proof: Note that

$$
\begin{aligned}
- & \nu_{1} h\left(S^{n} \mid W\right)-\nu_{2}\left(h\left(S^{n}+Z_{2}^{n} \mid W\right)-h\left(S^{n}+Z_{1}^{n} \mid W\right)\right) \\
= & \nu_{1}\left(h\left(S^{n}+Z_{1}^{n} \mid W\right)-h\left(S^{n} \mid W\right)\right)-\nu_{2} h\left(S^{n}+Z_{2}^{n} \mid W\right) \\
& -\left(\nu_{1}-\nu_{2}\right) h\left(S^{n}+Z_{1}^{n} \mid W\right) .
\end{aligned}
$$

Since $\sigma_{S^{n}+Z_{i}^{n} \mid W}^{2}=\sigma_{S^{n \mid} W}^{2}+\sigma_{Z_{i}}^{2} \leq d+\sigma_{Z_{i}}^{2}, i=1,2$, it follows from Lemma 7 that

$$
\begin{aligned}
& -\nu_{2} h\left(S^{n}+Z_{2}^{n} \mid W\right)-\left(\nu_{1}-\nu_{2}\right) h\left(S^{n}+Z_{1}^{n} \mid W\right) \\
\geq & -\frac{\nu_{2} n}{2} \log \left(2 \pi e\left(d+\sigma_{Z_{2}}^{2}\right)\right) \\
& -\frac{\left(\nu_{1}-\nu_{2}\right) n}{2} \log \left(2 \pi e\left(d+\sigma_{Z_{1}}^{2}\right)\right) .
\end{aligned}
$$

Furthermore, by Lemma 8 , we have

$$
\nu_{1}\left(h\left(S^{n}+Z_{1}^{n} \mid W\right)-h\left(S^{n} \mid W\right)\right) \geq \frac{\nu_{1} n}{2} \log \left(\frac{d+\sigma_{Z_{1}}^{2}}{d}\right) .
$$

Substituting (63) and (64) into (62) completes the proof of Lemma 9.

Now we are ready to prove Theorem 5 . It can be verified that

1) if $\rho_{1}=\rho_{2}=0$, then Theorem 5 is implied by Lemma 7;

2) if $\rho_{1} \neq \rho_{2}=0$ or $\sigma_{N_{2}}^{2}=\sigma_{N_{3}}^{2}$, then Theorem 5 is implied by Lemma 8

3) if $\rho_{2} \neq \rho_{1}=0$, then Theorem 5 is implied by Lemma 9 . 
Therefore, it suffices to consider the case where $\sigma_{N_{2}}^{2}<\sigma_{N_{3}}^{2}$ and $\rho_{i} \neq 0, i=1,2$. With no loss of generality, we shall assume $\rho_{1}=\rho_{2}=1$.

First consider the case $\sigma_{N_{1}}^{2} \geq \sigma_{N_{3}}^{2}$. Note that

$$
\begin{aligned}
& \nu_{1}\left(h\left(S^{n}+N_{1}^{n} \mid W\right)-h\left(S^{n} \mid W\right)\right) \\
& \quad-\nu_{2}\left(h\left(S^{n}+N_{3}^{n} \mid W\right)-h\left(S^{n}+N_{2}^{n} \mid W\right)\right) \\
& =\nu_{1}\left(h\left(S^{n}+N_{1}^{n} \mid W\right)-h\left(S^{n}+N_{3}^{n} \mid W\right)\right) \\
& \quad+\nu_{2}\left(h\left(S^{n}+N_{2}^{n} \mid W\right)-h\left(S^{n} \mid W\right)\right) \\
& \quad+\left(\nu_{1}-\nu_{2}\right)\left(h\left(S^{n}+N_{3}^{n} \mid W\right)-h\left(S^{n} \mid W\right)\right) .
\end{aligned}
$$

By Lemma 8

$$
\begin{aligned}
& \nu_{2}\left(h\left(S^{n}+N_{2}^{n} \mid W\right)-h\left(S^{n} \mid W\right)\right) \\
& \quad+\left(\nu_{1}-\nu_{2}\right)\left(h\left(S^{n}+N_{3}^{n} \mid W\right)-h\left(S^{n} \mid W\right)\right) \\
& \geq \frac{\nu_{2} n}{2} \log \left(\frac{d+\sigma_{N_{2}}^{2}}{d}\right)+\frac{\left(\nu_{1}-\nu_{2}\right) n}{2} \log \left(\frac{d+\sigma_{N_{3}}^{2}}{d}\right) .
\end{aligned}
$$

If $\sigma_{N_{1}}^{2}>\sigma_{N_{3}}^{2}$, then without loss of generality, we can assume $N_{1}^{n}=N_{3}^{n}+\Theta^{n}$, where $\Theta^{n}$ is independent of $\left(N_{3}^{n}, S^{n}, W\right)$, and the entries of $\Theta^{n}$ are i.i.d. Gaussian random variables with mean zero and variance $\sigma_{N_{1}}^{2}-\sigma_{N_{3}}^{2}$. Hence

$$
\begin{aligned}
& \nu_{1}\left(h\left(S^{n}+N_{1}^{n} \mid W\right)-h\left(S^{n}+N_{3}^{n} \mid W\right)\right) \\
& =\nu_{1}\left(h\left(S^{n}+N_{3}^{n}+\Theta^{n} \mid W\right)-h\left(S^{n}+N_{3}^{n} \mid W\right)\right) \\
& \geq \frac{\nu_{1} n}{2} \log \left(\frac{d+\sigma_{N_{1}}^{2}}{d+\sigma_{N_{3}}^{2}}\right)
\end{aligned}
$$

where (67) follows from Lemma 8 and the fact that $\sigma_{S^{n}+N_{3}^{n} \mid W}^{2}=\sigma_{S^{n} \mid W}^{2}+\sigma_{N_{3}}^{2} \leq d+\sigma_{N_{3}}^{2}$. It is clear that (67) also holds when $\sigma_{N_{1}}^{2}=\sigma_{N_{3}}^{2}$. Substituting (66) and (67) into (65) gives

$$
\begin{aligned}
& \nu_{1}\left(h\left(S^{n}+N_{1}^{n} \mid W\right)-h\left(S^{n} \mid W\right)\right) \\
& \quad-\nu_{2}\left(h\left(S^{n}+N_{3}^{n} \mid W\right)-h\left(S^{n}+N_{2}^{n} \mid W\right)\right) \\
& \geq \frac{\nu_{1} n}{2} \log \left(\frac{d+\sigma_{N_{1}}^{2}}{d}\right)-\frac{\nu_{2} n}{2} \log \left(\frac{d+\sigma_{N_{3}}^{2}}{d+\sigma_{N_{2}}^{2}}\right)
\end{aligned}
$$

which is the desired result.

Now it suffices to prove Theorem 5 for the case $\sigma_{N_{1}}^{2}<\sigma_{N_{3}}^{2}$. To this end, we use a reduction method inspired by [18]. Without loss of generality, we assume $N_{3}^{n}=N_{i}^{n}+\Theta_{i}^{n}$, where $\Theta_{i}^{n}$ is independent of $\left(N_{i}^{n}, S^{n}, W\right)$, and the entries of $\Theta_{i}^{n}$ are i.i.d. Gaussian random variables with mean zero and variance $\sigma_{N_{3}}^{2}-$ $\sigma_{N_{i}}^{2}, i=1,2$. Note that

$$
\begin{aligned}
\nu_{1}( & \left.h\left(S^{n}+N_{1}^{n} \mid W\right)-h\left(S^{n} \mid W\right)\right) \\
& -\nu_{2}\left(h\left(S^{n}+N_{3}^{n} \mid W\right)-h\left(S^{n}+N_{2}^{n} \mid W\right)\right) \\
= & \nu_{1}\left(h\left(S^{n}+N_{3}^{n} \mid W\right)+h\left(S^{n}+N_{1}^{n} \mid S^{n}+N_{3}^{n}, W\right)\right. \\
& \left.\quad-h\left(S^{n}+N_{3}^{n} \mid S^{n}+N_{1}^{n}, W\right)\right) \\
- & \nu_{1}\left(h\left(S^{n}+N_{3}^{n} \mid W\right)+h\left(S^{n} \mid S^{n}+N_{3}^{n}, W\right)\right. \\
& \left.-h\left(S^{n}+N_{3}^{n} \mid S^{n}, W\right)\right)-\nu_{2} h\left(S^{n}+N_{3}^{n} \mid W\right) \\
& +\nu_{2}\left(h\left(S^{n}+N_{3}^{n} \mid W\right)+h\left(S^{n}+N_{2}^{n} \mid S^{n}+N_{3}^{n}, W\right)\right. \\
& \left.-h\left(S^{n}+N_{3}^{n} \mid S^{n}+N_{2}^{n}, W\right)\right) \\
= & \nu_{1}\left(h\left(S^{n}+N_{1}^{n} \mid S^{n}+N_{3}^{n}, W\right)-h\left(\Theta_{1}^{n}\right)\right)
\end{aligned}
$$

$$
\begin{aligned}
& -\nu_{1}\left(h\left(S^{n} \mid S^{n}+N_{3}^{n}, W\right)-h\left(N_{3}^{n}\right)\right) \\
+ & \nu_{2}\left(h\left(S^{n}+N_{2}^{n} \mid S^{n}+N_{3}^{n}, W\right)-h\left(\Theta_{2}^{n}\right)\right) \\
= & \nu_{1}\left(h\left(S^{n}+N_{1}^{n} \mid S^{n}+N_{3}^{n}, W\right)\right. \\
& \left.\quad-\frac{n}{2} \log \left(2 \pi e\left(\sigma_{N_{3}}^{2}-\sigma_{N_{1}}^{2}\right)\right)\right) \\
& -\nu_{1}\left(h\left(S^{n} \mid S^{n}+N_{3}^{n}, W\right)-\frac{n}{2} \log \left(2 \pi e \sigma_{N_{3}}^{2}\right)\right) \\
+ & \nu_{2}\left(h\left(S^{n}+N_{2}^{n} \mid S^{n}+N_{3}^{n}, W\right)\right. \\
& \left.-\frac{n}{2} \log \left(2 \pi e\left(\sigma_{N_{3}}^{2}-\sigma_{N_{2}}^{2}\right)\right)\right) .
\end{aligned}
$$

Let $Q_{i}^{n}=N_{i}^{n}-\mathbb{E}\left[N_{i}^{n} \mid N_{3}^{n}\right], i=1,2$. It can be verified that $Q_{i}^{n}=N_{i}^{n}-\sigma_{N_{i}}^{2} \sigma_{N_{3}}^{-2} N_{3}^{n}, i=1,2$. Moreover, it is clear that $Q_{i}^{n}$ is independent of $\left(N_{3}^{n}, S^{n}, W\right)$, and the entries of $Q_{i}^{n}$ are i.i.d. Gaussian random variables with mean zero and variance $\sigma_{N_{i}}^{2}-\sigma_{N_{i}}^{4} \sigma_{N_{3}}^{-2}, i=1,2$. Note that

$$
\begin{aligned}
& h\left(S^{n}+N_{i}^{n} \mid S^{n}+N_{3}^{n}, W\right) \\
& =h\left(S^{n}+\sigma_{N_{i}}^{2} \sigma_{N_{3}}^{-2} N_{3}^{n}+Q_{i}^{n} \mid S^{n}+N_{3}^{n}, W\right) \\
& =h\left(\left(1-\sigma_{N_{i}}^{2} \sigma_{N_{3}}^{-2}\right) S^{n}+Q_{i}^{n} \mid S^{n}+N_{3}^{n}, W\right) \\
& \geq \frac{n}{2} \log \left(e^{\frac{2}{n} h\left(\left(1-\sigma_{N_{i}}^{2} \sigma_{N_{3}}^{-2}\right) S^{n} \mid S^{n}+N_{3}^{n}, W\right)}+e^{\frac{2}{n} h\left(Q_{i}^{n}\right)}\right) \\
& =\frac{n}{2} \log \left(\left(1-\sigma_{N_{i}}^{2} \sigma_{N_{3}}^{-2}\right)^{2} e^{\frac{2}{n} h\left(S^{n} \mid S^{n}+N_{3}^{n}, W\right)}\right. \\
& \left.\quad \quad+2 \pi e\left(\sigma_{N_{i}}^{2}-\sigma_{N_{i}}^{4} \sigma_{N_{3}}^{-2}\right)\right), \quad i=1,2
\end{aligned}
$$

where (69) follows by the entropy power inequality. Substituting (70) into (68), we obtain

$$
\begin{aligned}
& \nu_{1}\left(h\left(S^{n}+N_{1}^{n} \mid W\right)-h\left(S^{n} \mid W\right)\right) \\
& -\nu_{2}\left(h\left(S^{n}+N_{3}^{n} \mid W\right)-h\left(S^{n}+N_{2}^{n} \mid W\right)\right) \\
& \geq \nu_{1}\left(\frac { n } { 2 } \operatorname { l o g } \left(\left(1-\sigma_{N_{1}}^{2} \sigma_{N_{3}}^{-2}\right)^{2} e^{\frac{2}{n} h\left(S^{n} \mid S^{n}+N_{3}^{n}, W\right)}\right.\right. \\
& \left.\left.+2 \pi e\left(\sigma_{N_{1}}^{2}-\sigma_{N_{1}}^{4} \sigma_{N_{3}}^{-2}\right)\right)-\frac{n}{2} \log \left(2 \pi e\left(\sigma_{N_{3}}^{2}-\sigma_{N_{1}}^{2}\right)\right)\right) \\
& -\nu_{1}\left(h\left(S^{n} \mid S^{n}+N_{3}^{n}, W\right)-\frac{n}{2} \log \left(2 \pi e \sigma_{N_{3}}^{2}\right)\right) \\
& +\nu_{2}\left(\frac { n } { 2 } \operatorname { l o g } \left(\left(1-\sigma_{N_{2}}^{2} \sigma_{N_{3}}^{-2}\right)^{2} e^{\frac{2}{n} h\left(S^{n} \mid S^{n}+N_{3}^{n} \cdot W\right)}\right.\right. \\
& \left.\left.+2 \pi e\left(\sigma_{N_{2}}^{2}-\sigma_{N_{2}}^{4} \sigma_{N_{3}}^{-2}\right)\right)-\frac{n}{2} \log \left(2 \pi e\left(\sigma_{N_{3}}^{2}-\sigma_{N_{2}}^{2}\right)\right)\right) .
\end{aligned}
$$

Now we proceed to bound $h\left(S^{n} \mid S^{n}+N_{3}^{n}, W\right)$. Let $\hat{S}^{n}$ be an estimate of $S^{n}$ based on $\left(S^{n}+N_{3}^{n}, W\right)$, where

$$
\begin{aligned}
\hat{S}^{n}= & \sigma_{S^{n} \mid W}^{2}\left(\sigma_{S^{n} \mid W}^{2}+\sigma_{N_{3}}^{2}\right)^{-1}\left(S^{n}-\mathbb{E}\left[S^{n} \mid W\right]+N_{3}^{n}\right) \\
& +\mathbb{E}\left[S^{n} \mid W\right] .
\end{aligned}
$$

It can be verified that

$$
\frac{1}{n} \mathbb{E}\left[\left(S^{n}-\hat{S}^{n}\right)\left(S^{n}-\hat{S}^{n}\right)^{T}\right]=\frac{\sigma_{S^{n} \mid W}^{2} \sigma_{N_{3}}^{2}}{\sigma_{S^{n} \mid W}^{2}+\sigma_{N_{3}}^{2}} .
$$

Since $\sigma_{S^{n} \mid S^{n}+N_{3}^{n}, W}^{2} \leq \frac{1}{n} \mathbb{E}\left[\left(S^{n}-\hat{S}^{n}\right)\left(S^{n}-\hat{S}^{n}\right)^{T}\right]$ and $\sigma_{S^{n} \mid W}^{2} \leq d$, we have

$$
\sigma_{S^{n} \mid S^{n}+N_{3}^{n}, W}^{2} \leq\left(d^{-1}+\sigma_{N_{3}}^{-2}\right)^{-1}
$$


which, by Lemma 7, implies that

$$
h\left(S^{n} \mid S^{n}+N_{3}^{n}, W\right) \leq \frac{n}{2} \log \left(2 \pi e\left(d^{-1}+\sigma_{N_{3}}^{-2}\right)^{-1}\right) .
$$

In view of (71) and (72), we have

$$
\begin{aligned}
& \nu_{1}\left(h\left(S^{n}+N_{1}^{n} \mid W\right)-h\left(S^{n} \mid W\right)\right) \\
& \quad-\nu_{2}\left(h\left(S^{n}+N_{3}^{n} \mid W\right)-h\left(S^{n}+N_{2}^{n} \mid W\right)\right) \\
& \geq \min _{\tilde{\gamma} \in\left[0,\left(d^{-1}+\sigma_{N_{3}}^{-2}\right)^{-1}\right]} \nu_{1}\left(\frac { n } { 2 } \operatorname { l o g } \left(2 \pi e\left(1-\sigma_{N_{1}}^{2} \sigma_{N_{3}}^{-2}\right)^{2} \tilde{\gamma}\right.\right. \\
& \left.\left.\quad+2 \pi e\left(\sigma_{N_{1}}^{2}-\sigma_{N_{1}}^{4} \sigma_{N_{3}}^{-2}\right)\right)-\frac{n}{2} \log \left(2 \pi e\left(\sigma_{N_{3}}^{2}-\sigma_{N_{1}}^{2}\right)\right)\right) \\
& \quad-\nu_{1}\left(\frac{n}{2} \log (2 \pi e \tilde{\gamma})-\frac{n}{2} \log \left(2 \pi e \sigma_{N_{3}}^{2}\right)\right) \\
& +\nu_{2}\left(\frac { n } { 2 } \operatorname { l o g } \left(2 \pi e\left(1-\sigma_{N_{2}}^{2} \sigma_{N_{3}}^{-2}\right)^{2} \tilde{\gamma}\right.\right. \\
& \left.\left.\quad+2 \pi e\left(\sigma_{N_{2}}^{2}-\sigma_{N_{2}}^{4} \sigma_{N_{3}}^{-2}\right)\right)-\frac{n}{2} \log \left(2 \pi e\left(\sigma_{N_{3}}^{2}-\sigma_{N_{2}}^{2}\right)\right)\right) .
\end{aligned}
$$

Let $\gamma=\frac{\tilde{\gamma} \sigma_{N_{3}}^{2}}{\sigma_{N_{3}}^{2}-\tilde{\gamma}}$. Note that there is a one-to-one correspondence between $\gamma \in[0, d]$ and $\tilde{\gamma} \in\left[0,\left(d^{-1}+\sigma_{N_{3}}^{-2}\right)^{-1}\right]$. Moreover, it can be verified that

$$
\begin{aligned}
\nu_{1}( & \frac{n}{2} \log \left(2 \pi e\left(1-\sigma_{N_{1}}^{2} \sigma_{N_{3}}^{-2}\right)^{2} \tilde{\gamma}+2 \pi e\left(\sigma_{N_{1}}^{2}-\sigma_{N_{1}}^{4} \sigma_{N_{3}}^{-2}\right)\right) \\
& \left.-\frac{n}{2} \log \left(2 \pi e\left(\sigma_{N_{3}}^{2}-\sigma_{N_{1}}^{2}\right)\right)\right) \\
- & \nu_{1}\left(\frac{n}{2} \log (2 \pi e \tilde{\gamma})-\frac{n}{2} \log \left(2 \pi e \sigma_{N_{3}}^{2}\right)\right) \\
+ & \nu_{2}\left(\frac { n } { 2 } \operatorname { l o g } \left(2 \pi e\left(1-\sigma_{N_{2}}^{2} \sigma_{N_{3}}^{-2}\right)^{2} \tilde{\gamma}\right.\right. \\
& \left.\left.+2 \pi e\left(\sigma_{N_{2}}^{2}-\sigma_{N_{2}}^{4} \sigma_{N_{3}}^{-2}\right)\right)-\frac{n}{2} \log \left(2 \pi e\left(\sigma_{N_{3}}^{2}-\sigma_{N_{2}}^{2}\right)\right)\right) \\
= & \frac{\nu_{1} n}{2} \log \left(\frac{\gamma+\sigma_{N_{1}}^{2}}{\gamma}\right)-\frac{\nu_{2} n}{2}\left(\frac{\gamma+\sigma_{N_{3}}^{2}}{\gamma+\sigma_{N_{2}}^{2}}\right)
\end{aligned}
$$

which completes the proof.

\section{APPENDIX B}

\section{Proof of LeMma 3}

Consider $L$ discrete memoryless sources $X_{1}, \ldots, X_{L}$ with joint probability mass function $p\left(x_{1}, \ldots, x_{L}\right)$. Let $w_{i}(\cdot, \cdot)$ be a bounded distortion measure on $\mathcal{X}_{i} \times \hat{\mathcal{X}}_{i}$, where both $\mathcal{X}_{i}$ and $\hat{\mathcal{X}}_{i}$ are finite, $i=1, \ldots, L$. We shall show that if there exist auxiliary random variables $U_{i}$ (over finite alphabet $\left.\mathcal{U}_{i}\right), i=$ $1, \ldots, L$, and functions $\tilde{g}_{i}: \mathcal{U}_{1} \times \cdots \times \mathcal{U}_{i} \rightarrow \hat{\mathcal{X}}_{i}, i=1, \ldots, L$, and $\hat{g}_{i}: \mathcal{U}_{i} \rightarrow \hat{\mathcal{X}}_{i}, i=2, \ldots, L$, such that

P1) $\left(X_{2}, \ldots, X_{L}\right) \leftrightarrow X_{1} \leftrightarrow U_{1}$ form a Markov chain, and $\left(X_{j}\right)_{j \neq i} \leftrightarrow\left(X_{i}, U_{1}, \ldots, U_{i-1}\right) \leftrightarrow U_{i}$ form a Markov chain, $i=1, \ldots, L$,

P2) $\mathbb{E}\left[w_{i}\left(X_{i}, \tilde{g}_{i}\left(U_{1}, \ldots, U_{i}\right)\right)\right] \leq d_{i}, i=1, \ldots, L$, and $\mathbb{E}\left[w_{i}\left(X_{i}, \hat{g}_{i}\left(U_{i}\right)\right)\right] \leq \delta_{i}, i=2, \ldots, L$,

then $\bar{R} \in \mathcal{R}_{P}(\bar{d}, \bar{\delta})$ for any $\bar{R}$ satisfying

$$
\begin{aligned}
& R_{1} \geq I\left(X_{1} ; U_{1}\right), \\
& R_{i} \geq I\left(X_{i}, U_{1}, \ldots, U_{i-1} ; U_{i}\right), \quad i=2, \ldots, L .
\end{aligned}
$$

One can readily extend this result to the quadratic Gaussian case via a discretization procedure and certain limiting arguments [19].

As the proof is based on the standard techniques in network information theory, we only give a sketch here. We adopt the notation in [19].

Codebook Generation: Fix a conditional probability mass function $p\left(u_{1}, \ldots, u_{L} \mid x_{1}, \ldots, x_{L}\right)$ and functions $\tilde{g}_{i}: \mathcal{U}_{1} \times \cdots \times$ $\mathcal{U}_{i} \rightarrow \hat{\mathcal{X}}_{i}, i=1, \ldots, L$, and $\hat{g}_{i}: \mathcal{U}_{i} \rightarrow \hat{\mathcal{X}}_{i}, i=2, \ldots, L$, such that P1) and P2) are satisfied. Note that P1) is satisfied if $p\left(u_{1}, \ldots, u_{L} \mid x_{1}, \ldots, x_{L}\right)$ factors as

$$
\begin{aligned}
& p\left(u_{1}, \ldots, u_{L} \mid x_{1}, \ldots, x_{L}\right) \\
& =p\left(u_{1} \mid x_{1}\right) \prod_{i=2}^{L} p\left(u_{i} \mid x_{i}, u_{1}, \ldots, u_{i-1}\right) .
\end{aligned}
$$

For $i=1, \ldots, L$, randomly and independently generate $e^{n R_{i}}$ sequences $u_{i}^{n}\left(c_{i}\right), c_{i} \in\left[1: e^{n R_{i}}\right]$, each according to $\prod_{j=1}^{n} p_{U_{i}}\left(u_{i j}\right)$. The codebook is revealed to the encoders and the decoder.

Encoding: Given $x_{1}^{n}$, Encoder 1 finds an index $c_{1} \in[1$ : $\left.e^{n R_{1}}\right]$ such that $\left(x_{1}^{n}, u_{1}^{n}\left(c_{1}\right)\right) \in \mathcal{T}_{\epsilon_{1}}^{(n)}$; if there is more than one such index, it picks the smallest one among them; if there is no such index, it sets $c_{1}=1$. For $i=2, \ldots, L$, given $\left(x_{i}^{n}, c_{1}, \ldots, c_{i-1}\right)$, Encoder $i$ finds an index $c_{i} \in\left[1: e^{n R_{i}}\right]$ such that $\left(x_{i}^{n}, u_{1}^{n}\left(c_{1}\right), \ldots, u_{i}^{n}\left(c_{i}\right)\right) \in \mathcal{T}_{\epsilon_{i}}^{(n)}$; if there is more than one such index, it picks the smallest one among them; if there is no such index, it sets $c_{i}=1$. Here, we assume that $\epsilon_{L}>\cdots>\epsilon_{1}>0$. The indices $c_{1}, \ldots, c_{L}$ are then sent to the decoder.

Decoding: For $i=1, \ldots, L$, given $\left(c_{1}, \ldots, c_{i}\right)$, the decoder computes $\tilde{x}_{i j} \triangleq \tilde{g}_{i}\left(u_{1 j}\left(c_{1}\right), \ldots, u_{i j}\left(c_{i}\right)\right), j=1, \ldots, n$, and uses $\tilde{x}_{i}^{n}$ as the reconstruction of $x_{i}^{n}$. For $i=2, \ldots, L$, given $c_{i}$, the decoder computes $\hat{x}_{i j} \triangleq \hat{g}_{i}\left(u_{i j}\left(c_{i}\right)\right), j=1, \ldots, n$, and uses $\hat{x}_{i}^{n}$ as the reconstruction of $x_{i}^{n}$.

Error Analysis: Let $C_{i}$ denote the output of Encoder $i, i=1, \ldots, L$. By the covering lemma [19, Lemma 3.3, p. 62], $\mathbb{P}\left\{\left(X_{1}^{n}, U_{1}^{n}\left(C_{1}\right)\right) \in \mathcal{T}_{\epsilon_{1}}^{(n)}\right\}$ tends to one as $n \rightarrow \infty$ if $R_{1}>I\left(X_{1} ; U_{1}\right)+\delta_{1}\left(\epsilon_{1}\right)$, where $\delta_{1}\left(\epsilon_{1}\right)$ tends to zero as $\epsilon_{1} \rightarrow 0$. Then it follows from the conditional typicality lemma [19, p. 27] that $\mathbb{P}\left\{\left(X_{1}^{n}, \ldots, X_{L}^{n}, U_{1}^{n}\left(C_{1}\right)\right) \in \mathcal{T}_{\epsilon_{2}}^{(n)}\right\}$ tends to one as $n \rightarrow \infty$. For $i=2, \ldots, L$, by the covering lemma, $\mathbb{P}\left\{\left(X_{i}^{n}, U_{1}^{n}\left(C_{1}\right), \ldots, U_{i}^{n}\left(C_{i}\right)\right) \in \mathcal{T}_{\epsilon_{i}}^{(n)}\right\}$ tends to one as $n \rightarrow \infty$ if $R_{i}>I\left(X_{i}, U_{1}, \ldots, U_{i-1} ; U_{i}\right)+\delta_{i}\left(\epsilon_{i}\right)$, where $\delta_{i}\left(\epsilon_{i}\right)$ tends to zero as $\epsilon_{i} \rightarrow 0$; furthermore, it follows from [19, Lemma 12.3, p. 299] and the Markov lemma [19, Lemma 12.1, p. 296] that $\mathbb{P}\left\{\left(X_{1}^{n}, \ldots, X_{L}^{n}, U_{1}^{n}\left(C_{1}\right), \ldots, U_{i}^{n}\left(C_{i}\right)\right) \in \mathcal{T}_{\epsilon_{i+1}}^{(n)}\right\}$ tends to one as $n \rightarrow \infty$ if $\epsilon_{i}$ is sufficiently small compared to $\epsilon_{i+1}$. Therefore, for every $\epsilon>0$ and every $n \geq n(\epsilon)$ (with $n(\epsilon)$ determined by $\epsilon$ ), there exists a deterministic codebook conditioned on which the probability of $\left(X_{1}^{n}, \ldots, X_{L}^{n}, U_{1}^{n}\left(C_{1}\right), \ldots, U_{L}^{n}\left(C_{L}\right)\right) \notin \mathcal{T}_{\epsilon_{L+1}}^{(n)}$ is less than $\epsilon$. Now one can readily complete the proof by invoking the typical average lemma [19, p. 26]. 


\section{APPENDIX C}

PROOF OF LEMMA 5

Consider the following minimization problem:

$$
\min _{\gamma_{i} \in\left[0, d_{i}\right]} \phi\left(\gamma_{i}\right)
$$

where

$$
\begin{aligned}
\phi\left(\gamma_{i}\right)= & \frac{\mu_{i}}{2} \log \left(\frac{\left(\sigma_{X_{i}}^{2}-\theta_{i-1}\right) \gamma_{i}+\sigma_{X_{i}}^{2} \theta_{i-1}}{\gamma_{i}}\right) \\
& +\frac{\mu_{i+1}}{2} \log \left(\frac{\gamma_{i}+\sigma_{\Delta_{i}}^{2}}{\left(\sigma_{X_{i+1}}^{2}-\theta_{i}\right)\left(\gamma_{i}+\sigma_{\Delta_{i}}^{2}\right)+\sigma_{X_{i+1}}^{2} \theta_{i}}\right) .
\end{aligned}
$$

It is easy to verify that the objective function is a constant if $\theta_{i-1}=\theta_{i}=0$; moreover, the minimum in (73) is achieved at $\gamma_{i}=0$ if $\theta_{i-1}=0$ and $\theta_{i} \in\left(0, \sigma_{X_{i+1}}^{2}\right]$.

In the rest of the proof we shall assume $\theta_{i-1} \in\left(0, \sigma_{X_{i}}^{2}\right]$ (which implies that the minimum in (73) is not achieved at $\left.\gamma_{i}=0\right)$. Note that

$$
\frac{\partial \phi\left(\gamma_{i}\right)}{\partial \gamma_{i}}=\frac{1}{\hbar}\left(\tilde{a}_{i} \gamma_{i}^{2}+\tilde{b}_{i} \gamma_{i}+\tilde{c}_{i}\right)
$$

where $\tilde{a}_{i}, \tilde{b}_{i}$, and $\tilde{c}_{i}$ are defined in (32)-(34), respectively, and

$$
\begin{aligned}
\hbar= & 2\left(\left(\sigma_{X_{i}}^{2}-\theta_{i-1}\right) \gamma_{i}+\sigma_{X_{i}}^{2} \theta_{i-1}\right) \gamma_{i}\left(\gamma_{i}+\sigma_{\Delta_{i}}^{2}\right) \\
& \times\left(\left(\sigma_{X_{i+1}}^{2}-\theta_{i}\right)\left(\gamma_{i}+\sigma_{\Delta_{i}}^{2}\right)+\sigma_{X_{i+1}}^{2} \theta_{i}\right) .
\end{aligned}
$$

It is clear that $\tilde{b}_{i} \leq 0, \tilde{c}_{i}<0$, and $\hbar>0$ for $\gamma_{i} \in\left(0, d_{i}\right]$.

Now consider the following cases.

1) If $\tilde{a}_{i}>0$, then the equation

$$
\tilde{a}_{i} \gamma_{i}^{2}+\tilde{b}_{i} \gamma_{i}+\tilde{c}_{i}=0
$$

has a unique positive root at $\gamma_{i}=\hat{\gamma}_{i}$, where $\hat{\gamma}_{i}$ is defined in (36). We have $\frac{\partial \phi\left(\gamma_{i}\right)}{\partial \gamma_{i}}<0$ for $\gamma_{i} \in\left(0, \hat{\gamma}_{i}\right)$ and $\frac{\partial \phi\left(\gamma_{i}\right)}{\partial \gamma_{i}}>0$ for $\gamma_{i}>\hat{\gamma}_{i}$. As a consequence, the minimum in (73) is achieved at $\min \left\{\hat{\gamma}_{i}, d_{i}\right\}$.

2) If $\tilde{a}_{i} \leq 0$, we have $\frac{\partial \phi\left(\gamma_{i}\right)}{\partial \gamma_{i}}<0$ for $\gamma_{i}>0$. As a consequence, the minimum in (73) is achieved at $d_{i}$.

This completes the proof of Lemma 5.

\section{APPENDIX D}

\section{CONTINUITY OF $\tilde{\gamma}_{i^{*}}\left(\gamma_{i^{*}}^{*}\right)$}

To stress their dependence on $\left(\theta_{i^{*}-1}, \theta_{i^{*}}\right)$, we shall denote $\tilde{a}_{i^{*}}, \tilde{b}_{i^{*}}, \tilde{c}_{i^{*}}$, and $\hat{\gamma}_{i^{*}}$ by $\tilde{a}_{i^{*}}\left(\theta_{i^{*}-1}, \theta_{i^{*}}\right), \tilde{b}_{i^{*}}\left(\theta_{i^{*}-1}, \theta_{i^{*}}\right)$, $\tilde{c}_{i^{*}}\left(\theta_{i^{*}-1}, \theta_{i^{*}}\right)$, and $\hat{\gamma}_{i^{*}}\left(\theta_{i^{*}-1}, \theta_{i^{*}}\right)$ respectively. Define regions $\mathcal{R}_{1}$ and $\mathcal{R}_{2}$ as follows:

$$
\begin{aligned}
& \mathcal{R}_{1}=\left\{\left(\theta_{i^{*}-1}, \theta_{i^{*}}\right): \tilde{a}_{i^{*}}\left(\theta_{i^{*}-1}, \theta_{i^{*}}\right)>0\right\} \\
& \mathcal{R}_{2}=\left\{\left(\theta_{i^{*}-1}, \theta_{i^{*}}\right): \tilde{a}_{i^{*}}\left(\theta_{i^{*}-1}, \theta_{i^{*}}\right) \leq 0, \theta_{i^{*}-1} \in\left(0, \sigma_{X_{i^{*}}}^{2}\right]\right\} .
\end{aligned}
$$

It is clear that $\tilde{\gamma}_{i^{*}}\left(\gamma_{i^{*}}^{*}\right)$ varies continuously with $\gamma_{i^{*}}^{*}$ if $\left(\theta_{i^{*}-1}^{*}\left(\gamma_{i^{*}}^{*}\right), \theta_{i^{*}}^{*}\left(\gamma_{i^{*}}^{*}\right)\right)$ moves inside one of these two regions. Therefore, we only need to consider the case where $\left(\theta_{i^{*}-1}^{*}\left(\gamma_{i^{*}}^{*}\right), \theta_{i^{*}}^{*}\left(\gamma_{i^{*}}^{*}\right)\right)$ traverses through the boundary between $\mathcal{R}_{1}$ and $\mathcal{R}_{2}$.

Let $\left(\theta_{i^{*}-1}, \theta_{i^{*}}\right)$ be a boundary point between $\mathcal{R}_{1}$ and $\mathcal{R}_{2}$. It is clear that $\tilde{a}_{i^{*}}\left(\theta_{i^{*}-1}, \theta_{i^{*}}\right)=0$; moreover, it suffices to consider the case $\theta_{i^{*}-1}>0$ since we have $\theta_{i^{*}}=0$ if both $\tilde{a}_{i^{*}}\left(\theta_{i^{*}-1}, \theta_{i^{*}}\right)$ and $\theta_{i^{*}-1}$ are zero. As a consequence, we have $\left(\theta_{i^{*}-1}, \theta_{i^{*}}\right) \in \mathcal{R}_{2}$. Note that $\tilde{\gamma}_{i^{*}}\left(\gamma_{i^{*}}^{*}\right)=d_{i^{*}}$ if $\left(\theta_{i^{*}-1}^{*}\left(\gamma_{i^{*}}^{*}\right), \theta_{i^{*}}^{*}\left(\gamma_{i^{*}}^{*}\right)\right) \in \mathcal{R}_{2}$. On the other hand, as $\left(\theta_{i^{*}-1}^{*}\left(\gamma_{i^{*}}^{*}\right), \theta_{i^{*}}^{*}\left(\gamma_{i^{*}}^{*}\right)\right)$ moves toward $\left(\theta_{i^{*}-1}, \theta_{i^{*}}\right)$ from the $\mathcal{R}_{1}$ side, we have

$$
\begin{aligned}
& \tilde{a}_{i^{*}}\left(\theta_{i^{*}-1}^{*}\left(\gamma_{i^{*}}^{*}\right), \theta_{i^{*}}^{*}\left(\gamma_{i^{*}}^{*}\right)\right) \rightarrow 0 \\
& \tilde{c}_{i^{*}}\left(\theta_{i^{*}-1}^{*}\left(\gamma_{i^{*}}^{*}\right), \theta_{i^{*}}^{*}\left(\gamma_{i^{*}}^{*}\right)\right) \rightarrow \tilde{c}_{i^{*}}\left(\theta_{i^{*}-1}, \theta_{i^{*}}\right)>0 .
\end{aligned}
$$

Moreover, since

$$
\hat{\gamma}_{i^{*}}\left(\theta_{i^{*}-1}^{*}\left(\gamma_{i^{*}}^{*}\right), \theta_{i^{*}}^{*}\left(\gamma_{i^{*}}^{*}\right)\right) \geq \sqrt{\frac{-\tilde{c}_{i^{*}}\left(\theta_{i^{*}-1}^{*}\left(\gamma_{i^{*}}^{*}\right), \theta_{i^{*}}^{*}\left(\gamma_{i^{*}}^{*}\right)\right)}{\tilde{a}_{i^{*}}\left(\theta_{i^{*}-1}^{*}\left(\gamma_{i^{*}}^{*}\right), \theta_{i^{*}}^{*}\left(\gamma_{i^{*}}^{*}\right)\right)}}
$$

it follows that $\hat{\gamma}_{i^{*}}\left(\theta_{i^{*}-1}^{*}\left(\gamma_{i^{*}}^{*}\right), \theta_{i^{*}}^{*}\left(\gamma_{i^{*}}^{*}\right)\right) \rightarrow \infty$, which further implies that

$$
\tilde{\gamma}_{i^{*}}\left(\gamma_{i^{*}}^{*}\right) \triangleq \min \left\{\hat{\gamma}_{i^{*}}\left(\theta_{i^{*}-1}^{*}\left(\gamma_{i^{*}}^{*}\right), \theta_{i^{*}}^{*}\left(\gamma_{i^{*}}^{*}\right)\right), d_{i^{*}}\right\}=d_{i^{*}}
$$

when $\left(\theta_{i^{*}-1}^{*}\left(\gamma_{i^{*}}^{*}\right), \theta_{i^{*}}^{*}\left(\gamma_{i^{*}}^{*}\right)\right)$ is sufficiently close to $\left(\theta_{i^{*}-1}, \theta_{i^{*}}\right)$. Therefore, $\tilde{\gamma}_{i^{*}}\left(\gamma_{i^{*}}^{*}\right)$ varies continuously with $\gamma_{i^{*}}^{*}$ when $\left(\theta_{i^{*}-1}^{*}\left(\gamma_{i^{*}}^{*}\right), \theta_{i^{*}}^{*}\left(\gamma_{i^{*}}^{*}\right)\right)$ traverses through the boundary between $\mathcal{R}_{1}$ and $\mathcal{R}_{2}$.

APPENDIX E

PROOF OF THEOREM 7

First consider the case $\sigma_{N_{3}}^{2} \geq \sigma_{N_{1}}^{2}$. Without loss of generality, we can assume $N_{3}^{n}=N_{1}^{n}+\Theta^{n}$, where $\Theta^{n}$ is independent of $\left(N_{1}^{n}, S^{n}, W\right)$, and the entries of $\Theta^{n}$ are i.i.d. Gaussian random variables with mean zero and variance $\sigma_{N_{3}}^{2}-\sigma_{N_{1}}^{2}$. Note that

$$
\begin{aligned}
& h\left(S^{n}+N_{3}^{n} \mid W\right)-h\left(S^{n}+N_{1}^{n} \mid W\right) \\
& =h\left(S^{n}+N_{1}^{n}+\Theta^{n} \mid W\right)-h\left(S^{n}+N_{1}^{n} \mid W\right) \\
& =I\left(\Theta^{n} ; S^{n}+N_{1}^{n}+\Theta^{n} \mid W\right) \\
& \leq I\left(\Theta^{n} ; S^{n}+N_{1}^{n}+\Theta^{n}, W\right) \\
& \leq I\left(\Theta^{n} ; N_{1}^{n}+\Theta^{n}\right) \\
& =\frac{n}{2} \log \left(\frac{\sigma_{N_{3}}^{2}}{\sigma_{N_{1}}^{2}}\right)
\end{aligned}
$$

where (74) is due to the fact that $\Theta^{n} \leftrightarrow\left(N_{1}^{n}+\Theta^{n}\right) \leftrightarrow\left(S^{n}+\right.$ $\left.N_{1}^{n}+\Theta^{n}, W\right)$ form a Markov chain. Moreover, we have

$$
\begin{aligned}
h\left(S^{n}+N_{2}^{n} \mid W\right) & \geq h\left(S^{n}+N_{2}^{n} \mid S^{n}, W\right) \\
& =h\left(N_{2}^{n}\right) \\
& =\frac{n}{2} \log \left(2 \pi e \sigma_{N_{2}}^{2}\right) .
\end{aligned}
$$

As a consequence

$$
\begin{aligned}
& h\left(S^{n}+N_{3}^{n} \mid W\right)-h\left(S^{n}+N_{1}^{n} \mid W\right)-h\left(S^{n}+N_{2}^{n} \mid W\right) \\
& \leq \frac{n}{2} \log \left(2 \pi e \sigma_{N_{3}}^{2}\right)-\frac{n}{2} \log \left(2 \pi e \sigma_{N_{1}}^{2}\right)-\frac{n}{2} \log \left(2 \pi e \sigma_{N_{2}}^{2}\right)
\end{aligned}
$$


which is the desired result. By symmetry, this upper bound also holds when $\sigma_{N_{3}}^{2} \geq \sigma_{N_{2}}^{2}$.

Now consider the case $\sigma_{N_{3}}^{2}<\min \left\{\sigma_{N_{1}}^{2}, \sigma_{N_{2}}^{2}\right\}$. Without loss of generality, we assume $N_{i}^{n}=N_{3}^{n}+\Theta_{i}^{n}$, where $\Theta_{i}^{n}$ is independent of $\left(N_{3}^{n}, S^{n}, W\right)$, and the entries of $\Theta_{i}^{n}$ are i.i.d. Gaussian random variables with mean zero and variance $\sigma_{N_{i}}^{2}-\sigma_{N_{3}}^{2}, i=$ 1,2 . Note that

$$
\begin{aligned}
& h\left(S^{n}+N_{i}^{n} \mid W\right) \\
& =h\left(S^{n}+N_{3}^{n}+\Theta_{i}^{n} \mid W\right) \\
& \geq \frac{n}{2} \log \left(e^{\frac{2}{n} h\left(S^{n}+N_{3}^{n} \mid W\right)}+e^{\frac{2}{n} h\left(\Theta_{i}^{n}\right)}\right) \\
& =\frac{n}{2} \log \left(e^{\frac{2}{n} h\left(S^{n}+N_{3}^{n} \mid W\right)}+2 \pi e\left(\sigma_{N_{i}}^{2}-\sigma_{N_{3}}^{2}\right)\right), \quad i=1,2
\end{aligned}
$$

where (75) is due to the entropy power inequality. Note that $\sigma_{S^{n}+N_{i}^{n} \mid W}^{2}=\sigma_{S^{n} \mid W}^{2}+\sigma_{N_{i}}^{2} \leq d+\sigma_{N_{i}}^{2}, i=1,2$. Therefore, it follows from Lemma 7 in Appendix A that

$$
h\left(S^{n}+N_{i}^{n} \mid W\right) \leq \frac{n}{2} \log \left(2 \pi e\left(d+\sigma_{N_{i}}^{2}\right)\right), \quad i=1,2 .
$$

In view of (76) and (77), we have

$$
\begin{aligned}
& h\left(S^{n}+N_{3}^{n} \mid W\right)-h\left(S^{n}+N_{1}^{n} \mid W\right)-h\left(S^{n}+N_{2}^{n} \mid W\right) \\
& \leq \min _{\gamma \in[0, d]} \frac{n}{2} \log \left(2 \pi e\left(\gamma+\sigma_{N_{3}}^{2}\right)\right) \\
& \quad-\frac{n}{2} \log \left(2 \pi e\left(\gamma+\sigma_{N_{3}}^{2}\right)+2 \pi e\left(\sigma_{N_{1}}^{2}-\sigma_{N_{3}}^{2}\right)\right) \\
& \quad-\frac{n}{2} \log \left(2 \pi e\left(\gamma+\sigma_{N_{3}}^{2}\right)+2 \pi e\left(\sigma_{N_{2}}^{2}-\sigma_{N_{3}}^{2}\right)\right) \\
& =\min _{\gamma \in[0, d]} \frac{n}{2} \log \left(2 \pi e\left(\gamma+\sigma_{N_{3}}^{2}\right)\right)-\frac{n}{2} \log \left(2 \pi e\left(\gamma+\sigma_{N_{1}}^{2}\right)\right) \\
& \quad-\frac{n}{2} \log \left(2 \pi e\left(\gamma+\sigma_{N_{2}}^{2}\right)\right)
\end{aligned}
$$

which completes the proof.

\section{REFERENCES}

[1] H. Viswanathan and T. Berger, "Sequential coding of correlated sources," IEEE Trans. Inf. Theory, vol. 46, no. 1, pp. 236-246, Jan. 2000.

[2] H. S. Witsenhausen, "On the structure of real-time source coders," Bell Syst. Tech. J., vol. 58, no. 6, pp. 1437-1451, Jul.-Aug. 1979.

[3] N. Ma and P. Ishwar, "On delayed sequential coding of correlated sources," IEEE Trans. Inf. Theory, vol. 57, no. 6, pp. 3763-3782, Jun. 2011.

[4] E.-H. Yang, L. Zheng, D.-K. He, and Z. Zhang, "Rate distortion theory for causal video coding: Characterization, computation algorithm, and comparison," IEEE Trans. Inf. Theory, vol. 57, no. 8, pp. 5258-5280, Aug. 2011.

[5] J. Wang and X. Wu, "Information flows in video coding," in Proc. IEEE Data Comput. Conf., Snowbird, UT, USA, Mar. 24-26, 2010, pp. $149-158$.

[6] A. A. El Gamal and T. M. Cover, "Achievable rates for multiple descriptions," IEEE Trans. Inf. Theory, vol. IT-28, no. 6, pp. 851-857, Nov. 1982.

[7] J. Chen, "Rate region of Gaussian multiple description coding with individual and central distortion constraints," IEEE Trans. Inf. Theory, vol. 55, no. 9, pp. 3991-4005, Sep. 2009.

[8] L. Ozarow, "On a source coding problem with two channels and three receivers," Bell Syst. Tech. J., vol. 59, no. 10, pp. 1909-1921, Dec. 1980.

[9] H. Wang and P. Viswanath, "Vector Gaussian multiple description with individual and central receivers," IEEE Trans. Inf. Theory, vol. 53, no. 6, pp. 2133-2153, Jun. 2007.
[10] R. Zamir and M. Feder, "On universal quantization by randomized uniform/lattice quantizer," IEEE Trans. Inf. Theory, vol. 38, no. 2, pp. 428-436, Mar. 1992.

[11] R. Zamir and M. Feder, "On lattice quantization noise," IEEE Trans. Inf. Theory, vol. 42, no. 4, pp. 1152-1159, Jul. 1996.

[12] R. Zamir and M. Feder, "Information rates of pre/post-filtered dithered quantizers," IEEE Trans. Inf. Theory, vol. 42, no. 5, pp. 1340-1353, Sep. 1996.

[13] T. Liu and P. Viswanath, "An extremal inequality motivated by multiterminal information-theoretic problems," IEEE Trans. Inf. Theory, vol. 53, no. 5, pp. 1839-1851, May 2007.

[14] H. Weingarten, T. Liu, S. Shamai (Shitz), Y. Steinberg, and P. Viswanath, "The capacity region of the degraded multiple-input multiple-output compound broadcast channel," IEEE Trans. Inf. Theory, vol. 55, no. 11, pp. 5011-5023, Nov. 2009.

[15] R. Liu, T. Liu, H. V. Poor, and S. Shamai (Shitz), "A vector generalization of Costa's entropy-power inequality with applications," IEEE Trans. Inf. Theory, vol. 56, no. 4, pp. 1865-1879, Apr. 2010.

[16] S. Ihara, "On the capacity of channels with additive non-Gaussian noise," Inf. Control, vol. 37, no. 1, pp. 34-39, Apr. 1978.

[17] S. N. Diggavi and T. M. Cover, "The worst additive noise under a covariance constraint," IEEE Trans. Inf. Theory, vol. 47, no. 7, pp. 3072-3081, Nov. 2001.

[18] S. Watanabe and Y. Oohama, Secret key agreement from vector Gaussian sources by rate limited public communication [Online]. Available: http://arxiv.org/abs/1009.5760

[19] A. El Gamal and Y.-H. Kim, Network Information Theory. Cambridge, U.K.: Cambridge Univ. Press, 2011.

Lin Song received the B.Sc. degree in Electronic Information Engineering, the M.S. degree in Communication and Information Systems from Harbin Institute of Technology, Harbin, China, in 2006 and 2008, respectively, and the $\mathrm{Ph} . \mathrm{D}$. degree in Electrical and Computer Engineering from McMaster University, Hamilton, ON, Canada, in 2012. She is currently a postdoctoral research assistant at McMaster University, Hamilton, ON, Canada. Her research interests include information and coding theory.

Jun Chen (S'03-M'06) received the B.E. degree with honors in communication engineering from Shanghai Jiao Tong University, Shanghai, China, in 2001 and the M.S. and Ph.D. degrees in electrical and computer engineering from Cornell University, Ithaca, NY, in 2004 and 2006, respectively. He was a Postdoctoral Research Associate in the Coordinated Science Laboratory at the University of Illinois at Urbana-Champaign, Urbana, IL, from 2005 to 2006, and a Postdoctoral Fellow at the IBM Thomas J. Watson Research Center, Yorktown Heights, NY, from 2006 to 2007. He is currently an Assistant Professor of Electrical and Computer Engineering at McMaster University, Hamilton, ON, Canada, and holds the Barber-Gennum Chair in Information Technology. His research interests include information theory, wireless communications, and signal processing.

Dr. Chen received several awards for his research, including the Josef Raviv Memorial Postdoctoral Fellowship in 2006, the Early Researcher Award from the Province of Ontario in 2010, and the IBM Faculty Award in 2010.

Jia Wang received the B.Sc. degree in electronic engineering, the M.S. degree in pattern recognition and intelligence control, and the $\mathrm{Ph}$.D. degree in electronic engineering from Shanghai Jiao Tong University, China, in 1997, 1999, and 2002, respectively.

$\mathrm{He}$ is currently an Associate Professor of the Institute of Image Communication and Information Processing, Shanghai Jiao Tong University, and a member of Shanghai Key Laboratory of Digital Media Processing and Transmission. His research interests include multiuser information theory and its application in video coding.

Tie Liu received his B.S. (1998) and M.S. (2000) degrees, both in Electrical Engineering, from Tsinghua University, Beijing, China and a second M.S. degree in Mathematics (2004) and Ph.D. degree in Electrical and Computer Engineering (2006) from the University of Illinois at Urbana-Champaign. Since August 2006 he has been with Texas A\&M University, where he is currently an Associate Professor with the Department of Electrical and Computer Engineering. His primary research interest is in information theory, with applications drawn from wireless communication and distributed storage systems.

Dr. Liu is a recipient of the M. E. Van Valkenburg Graduate Research Award (2006) from the University of Illinois at Urbana-Champaign and the Faculty Early Career Development (CAREER) Award (2009) from the National Science Foundation. 